\title{
Biotechnology-derived chitosans with non-random patterns of acetylation differ from conventional chitosans in their properties and activities
}

Jasper Wattjes ${ }^{1,2, \#, \text { Sruthi Sreekumar }}{ }^{1,2,3, \#}$, Anna Niehues ${ }^{1, \#}$, Tamara Mengoni $^{1}$, Ana C. Mendes ${ }^{2}$, Edwin R. Morris ${ }^{4}$, Francisco M. Goycoolea ${ }^{1,3}$, Bruno M. Moerschbacher ${ }^{1, *}$

${ }^{1}$ Institute for Biology and Biotechnology of Plants, University of Münster, 48143 Münster, Germany

${ }^{2}$ National Food Institute, Technical University of Denmark, 2800 Kgs. Lyngby, Denmark

${ }^{3}$ School of Food Science and Nutrition, University of Leeds, LS2 9JT, Leeds, United Kingdom

${ }^{4}$ School of Food and Nutritional Sciences, University College Cork, Cork, Ireland

\# these authors contributed equally

* corresponding author

\begin{abstract}
Chitosans are versatile biopolymers with multiple biological activities and potential applications. They are linear copolymers of glucosamine and $\mathrm{N}$-acetylglucosamine defined by their degree of polymerization (DP), fraction of acetylation $\left(F_{\mathrm{A}}\right)$, and pattern of acetylation (PA). Technical chitosans produced chemically from chitin possess defined DP and $F_{A}$ but random PA, while enzymatically produced natural chitosans are likely to have non-random PA. This natural process has not been replicated using biotechnology because chitin de- $N$-acetylases do not efficiently deacetylate crystalline chitin. Here, we show that such enzymes can partially $N$-acetylate polyglucosamine in the presence of excess acetate, yielding chitosans with $F_{\mathrm{A}}$ up to 0.7 and an enzymedependent non-random PA. The biotech chitosans differ from technical chitosans both in terms of physicochemical and nanoscale solution properties and biological activities. As with synthetic block co-polymers, controlling the distribution of building blocks within the biopolymer chain will open a new dimension of chitosan research and exploitation.
\end{abstract}

\section{Introduction}

Chitosans are binary copolymers of hydrophobic $N$-acetylglucosamine (GlcNAc) and cationic glucosamine (GlcN) linked by $\beta-1,4$-glycosidic bonds. In nature, chitosans are prominently found in the specialized fungal cell walls formed during host tissue penetration ${ }^{1,2}$. In the laboratory, chitosans can form gels, films, sponges, and nanoparticles and have been shown to interact with partly polyanionic/partly hydrophobic biomolecules such as proteins, nucleic acids, mucins, and phospholipid membranes ${ }^{3,4}$. Both the physicochemical properties and biological activities of chitosans are directly influenced by their degree of polymerization (DP), molar fraction of acetylation $\left(F_{A}\right)$, and, possibly, pattern of acetylation $(P A)^{5}$. It is possible to synthesize chitosan with tight control of the DP and $F_{A}$ by the partial chemical de- $N$-acetylation of chitin or $N$-acetylation of polyglucosamine, so that the influence of these parameters can be studied in detail ${ }^{6-8}$. The structure-function relationships thus derived, summarized in the chitosan matrix ${ }^{5}$, have allowed the development of chitosan-based applications for food preservation and plant protection, exploiting the antimicrobial properties of chitosans and their ability to induce plant defense responses ${ }^{9,10}$. However, biomedically relevant activities of chitosans still remain poorly understood on a molecular or nanoscale level, so that the development of chitosan-based biomaterials for medical applications lags behind the presumed potential of these functional biopolymers, possibly because of the unknown role of PA in the interaction of chitosans with human or animal cells ${ }^{5}$. 
In contrast to DP and $F_{A}$, the PA is more difficult to analyze and control ${ }^{11}$. Initially, chitosans produced by the heterogeneous de- $N$-acetylation of chitin were thought to possess blocks of acetyl groups, whereas chitosans produced by homogeneous de-acetylation or $N$-acetylation were predicted to have a more random PA ${ }^{12-14}$. However, ${ }^{13} \mathrm{C}$ nuclear magnetic resonance (NMR) spectroscopy showed that dyad frequencies of commercially available chitosans do not differ from what is expected for random PA, regardless of the production method ${ }^{15,16}$. It has therefore been impossible to investigate the potential influence of acetylation patterns on the properties and activities of chitosan polymers, but theoretical considerations strongly suggest such an influence exists ${ }^{5}$. Importantly, natural chitosans are likely to possess non-random acetylation patterns due to their enzymatic route of synthesis.

One way in which PA could influence the biological activity of chitosans is via the sequence-dependent activity of chitosanolytic enzymes ${ }^{17,18}$. Partially acetylated chitosans can be depolymerized by both chitinases and chitosanases. The former are ubiquitous whereas the latter appear to be restricted to microorganisms. Both types of enzyme tend to have more or less pronounced sequence specificity because their substrate-binding cleft consists of multiple subsites, each binding a single monomeric subunit and showing a preference for either GlcN or GlcNAc ${ }^{19-21}$. The PA of the substrate therefore determines its position in the binding site, in turn specifying GICN or GlcNAc units at and near the reducing and non-reducing ends of the oligomeric products. The PA of the substrate and the subsite specificities and preferences of the enzyme therefore combine to determine how readily the polymer is degraded and the types of oligomers produced. This is important because the biological activity of chitosan polymers in a given tissue may be exerted by the polymer itself, such as by electrostatic interactions with the plasma membrane of a target cell ${ }^{6}$, or by enzymatic degradation products generated in situ, which may be recognized by specific receptors ${ }^{8,22-25}$. In both cases, the PA strongly influences the pharmacokinetics of the chitosan polymers.

Although chemically produced conventional chitosans feature a random PA, natural chitosans produced by chitin deacetylases (CDAs) may possess non-random acetylation patterns ${ }^{26-28}$. However, CDAs are almost inactive on native chitin polymers in vitro probably because the substrate is crystalline ${ }^{29,30}$. In contrast, CDAs do act on soluble chitosan polymers with a high $F_{\mathrm{A}}$, converting them to low- $F_{\mathrm{A}}$ chitosans ${ }^{30-32}$. Such chemo-enzymatically produced chitosans can exhibit non-random acetylation patterns ${ }^{27,28}$. Depending on which CDA is used, the PA ranges from Bernoullian randomness to a block-like or more regular distribution of acetyl groups, as confirmed by ${ }^{13} \mathrm{C}-\mathrm{NMR}$ dyad analysis and enzymatic mass spectrometry (EMS) fingerprinting ${ }^{27}$. However, the $P_{\Sigma}$ values did not differ greatly from 1 , the value expected for random PA ${ }^{15}$, because the high- $F_{A}$ chitosan polymer used as a substrate was produced chemically and thus had a random PA. Accordingly, the products also tended towards randomness, with $P_{\Sigma}$-values of $0.75-1.31^{27}$. To address this issue, we approached the problem from the opposite perspective, using CDAs in reverse to partially $N$-acetylate polyglucosamine in the presence of excess acetate as a means to retain their regioselectivity and generate chitosans with a specific PA, as shown previously for GlcN oligomers ${ }^{17,33,34}$. We then tested the biotech chitosans to determine their physicochemical properties and biological activities compared to conventional technical chitosans with random acetylation.

\section{Results}

\section{CDA can $\boldsymbol{N}$-acetylate polyglucosamine, yielding high- $\boldsymbol{F}_{\mathrm{A}}$ chitosans with non-random acetylation}

We tested four recombinant fungal CDAs to determine whether they would act in reverse on polyglucosamine, producing partially acetylated chitosan polymers in the presence of excess acetate (Fig. 1). This yielded chitosans with a $F_{\mathrm{A}}$ as high as 0.7 (Fig. S1) and the process could be kinetically controlled, giving us access to the full spectrum of soluble chitosan polymers (Fig. 1a). EMS fingerprinting revealed that the enzymatically and chemically $\mathrm{N}$-acetylated chitosans differed in terms of PA, with the former showing average GIcN and GIcNAc block sizes that deviated from random PA chitosans in different directions depending on which enzyme we used. Detailed analysis of the chitinosanase products clearly showed that chitosans with a similar $F_{A}$ produced by different enzymes showed significantly different properties, confirming that each enzyme generated chitosan 
polymers with a unique PA (Fig. 1b). The oligomeric products of PgtCDA most closely resembled those of the conventional random-PA chitosan, whereas the products of AnCDA and PesCDA resembled each other but clearly differed from those of the conventional chitosan, featuring larger oligomers that indicated block-wise rather than random acetylation. In contrast, the products of CnCDA4 were dominated by smaller oligomers, indicating regular rather than random acetylation. A comparative analysis of block size distributions revealed that each enzyme generated unique products that differed from the chemical control (Fig. S1). Interestingly, these differences were already visible at early time points in the $N$-acetylation reaction when the $F_{\mathrm{A}}$ was low. We scaled up the PesCDA reaction to yield sufficient product for ${ }^{13} \mathrm{C}-\mathrm{NMR}$ dyad analysis. We initially used a sample with a $F_{A}$ of 0.5 but were unable to dissolve it in $\mathrm{D}_{2} \mathrm{O}$ following treatment with $\mathrm{HNO}_{2}$. Unlike chemically $\mathrm{N}$-acetylated chitosan polymers with the same $F_{\mathrm{A}}{ }^{35}$, the enzymatically $N$-acetylated chitosan polymer could not be solubilized using a stoichiometric quantity of acid but required $5 \%$ acetic acid, again indicating a non-random PA. This issue was not encountered with an enzymatically (also using PesCDA) $N$-acetylated chitosan with a $F_{A}$ of 0.33 , for which ${ }^{13} \mathrm{C}-\mathrm{NMR}$ dyad analysis revealed a $P_{\Sigma}$ value of 0.3 , verifying the strong block-wise distribution of acetyl groups (Fig. 1c; Fig. S1). 

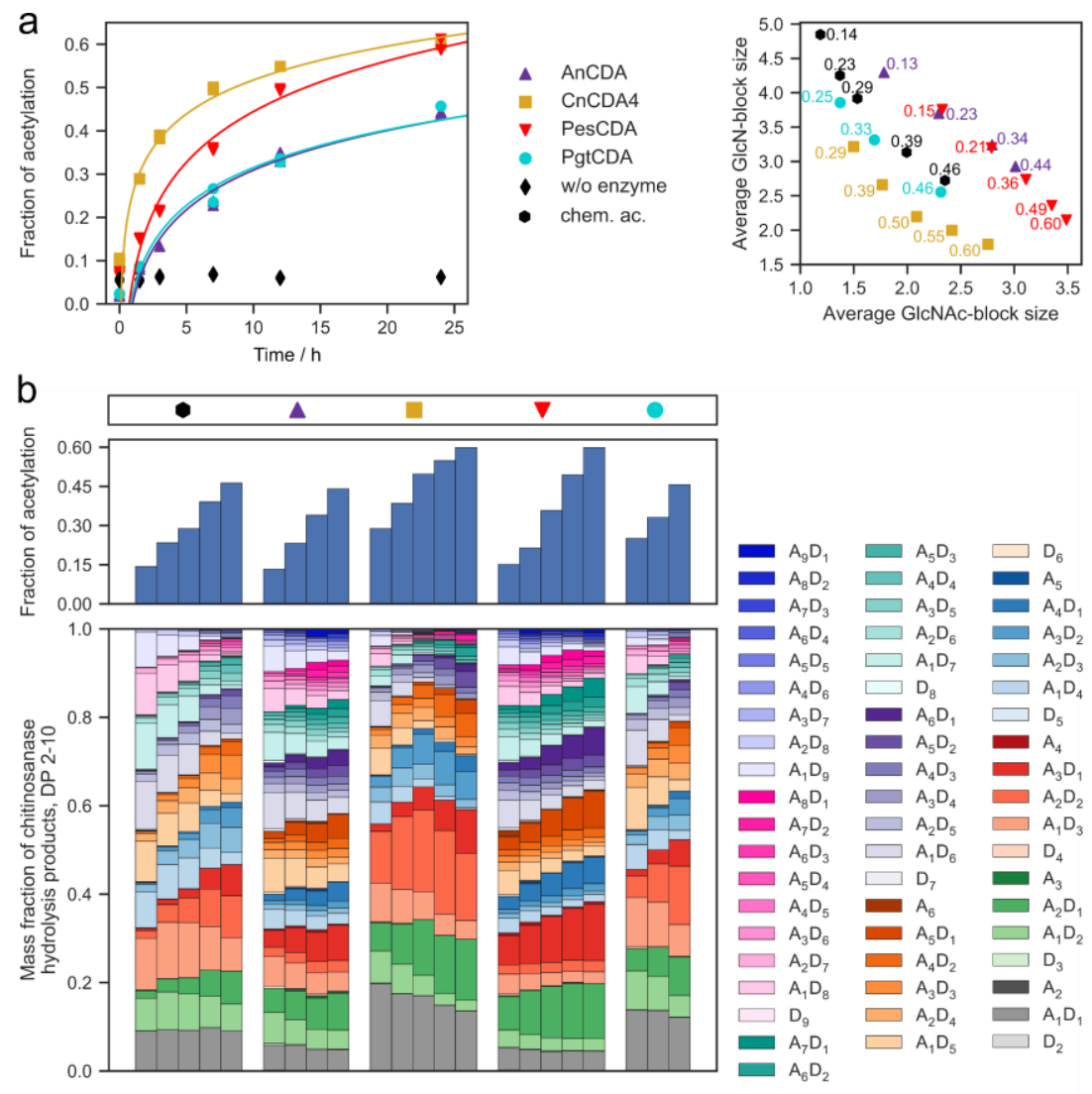

C

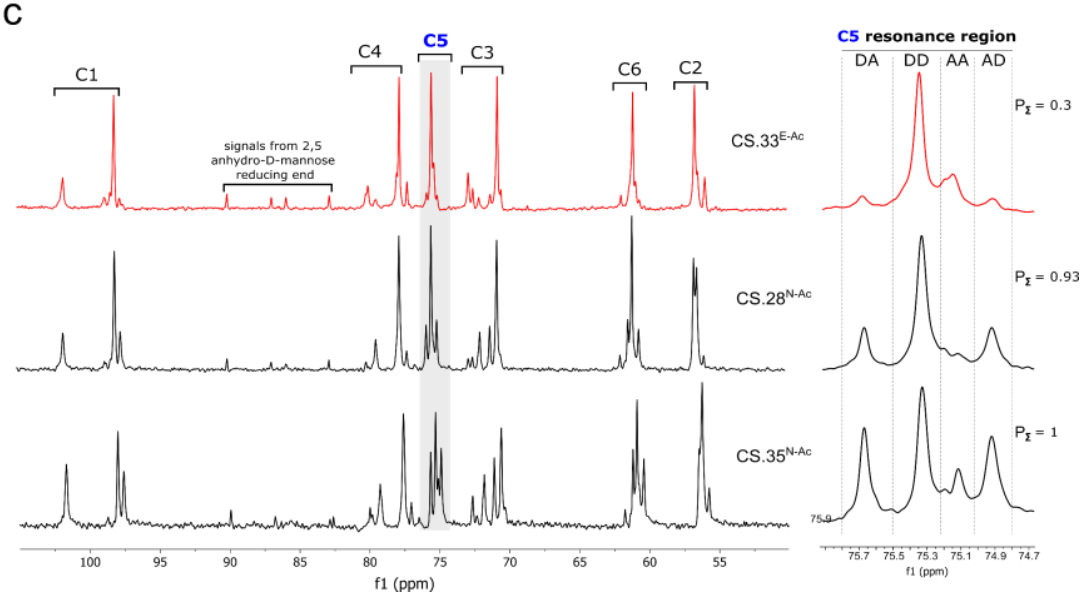

Fig. 1: CDAs can $N$-acetylate polyglucosamine, yielding high- $F_{\mathrm{A}}$ chitosans with non-random PA.

a) Polyglucosamine $\left(F_{\mathrm{A}}=0.03\right)$ was incubated in the presence of $1.5 \mathrm{M}$ sodium acetate for $24 \mathrm{~h}(\mathrm{pH} 7.5)$ with four different recombinant fungal CDAs (AnCDA from Aspergillus niger, CnCDA4 from Cryptococcus neoformans, PgtCDA from Puccinia graminis f. sp. tritici, and PesCDA from Pestalotiopsis sp.) or without enzyme as a control. The $F_{A}$ (left panel) and average block sizes of DP 2-10 (right panel) in the resulting chitosan polymers were analyzed using chitinosanase-based EMS fingerprinting. Chemically $N$-acetylated chitosans were used as positive controls. b) The chemically and enzymatically $N$-acetylated chitosans (see a) were hydrolyzed with chitinosanase, and products of DP 2-10 were analyzed by HILIC-ESI-MS to calculate the $F_{A}$ of the polymeric substrates (upper panel) and characterize the oligomeric products (lower panel). c) ${ }^{13} \mathrm{C}-\mathrm{NMR}$ analysis of one enzymatically (CS.33 ${ }^{\mathrm{E}-}$ Ac produced (using PesCDA) and two chemically $N$--acetylated chitosans with a similar $F_{A}\left(C S .28^{\mathrm{N}-A c}\right.$ and CS.35 $5^{\mathrm{N}-\mathrm{Ac}}$ ) (left panel) and magnification of the C-5 resonance region (right panel). The four dyad peak areas (IDA, IDD, IAA, and $\mathrm{I}_{\mathrm{AD}}$ ) were integrated and used to calculate $\mathrm{P}_{\Sigma}$ values. ${ }^{15}$ 


\section{The PA influences the physicochemical properties of chitosans in solution}

Next, we compared the physicochemical solution properties of the enzymatically $N$-acetylated $P_{\Sigma}=0.3$ block-PA chitosan polymer $\left(F_{\mathrm{A}}=0.33\right.$, $\mathrm{DP}=800$, dispersity $\left.(\Theta)=1.9\right)$ with those of a chemically $N$-acetylated $P_{\Sigma}=1.0$ random-PA chitosan $\left(F_{A}=0.34, D P=700, \emptyset=1.8\right.$ ) (Fig. 2). The intrinsic viscosity of the biotech chitosan in water was significantly lower than that of the conventional chitosan (Table S1). This difference was strongly reduced in the presence of $0.1 \mathrm{M} \mathrm{NaCl}$, indicating a more densely packed conformation in solution presumably caused by hydrophobic interactions between poly-GlcNAc blocks. This solution behavior was similar to that reported for a chemo-enzymatically prepared chitosan polymer with slightly more block-wise than random PA $\left(P_{\Sigma}=0.75\right)^{27}$. While the conventional chitosan readily formed nanoparticles by ionic gelation with tripolyphosphate (TPP) ${ }^{36,37}$, particle formation was limited with the biotech chitosan (Fig. 2a). The Z-average hydrodynamic diameter of the random-PA chitosan nanoparticles increased with the chitosan:TPP ratio, whereas that of the scarce block-PA chitosan nanoparticles remained surprisingly stable, again possibly reflecting hydrophobic interactions between poly-GlcNAc blocks which are not influenced by the molar charge ratio. In contrast, both types of chitosan readily yielded nanocapsules (chitosan-coated nanoemulsions ${ }^{38}$ with no significant differences between the two forms (Fig. 2b). Whereas chitosan forms the bulk of chitosan-TPP nanoparticles, the nanocapsules feature a thin chitosan surface layer, apparently with less influence on the overall properties of the system. We also found that both types of chitosan were able to form nanoparticles prepared using an electrospray technique (Fig. 2c) in the presence of $30 \%$ acetic acid and $30 \%$ ethanol 39,40 . These conditions are likely to reduce the influence of electrostatic and hydrophobic interactions. 

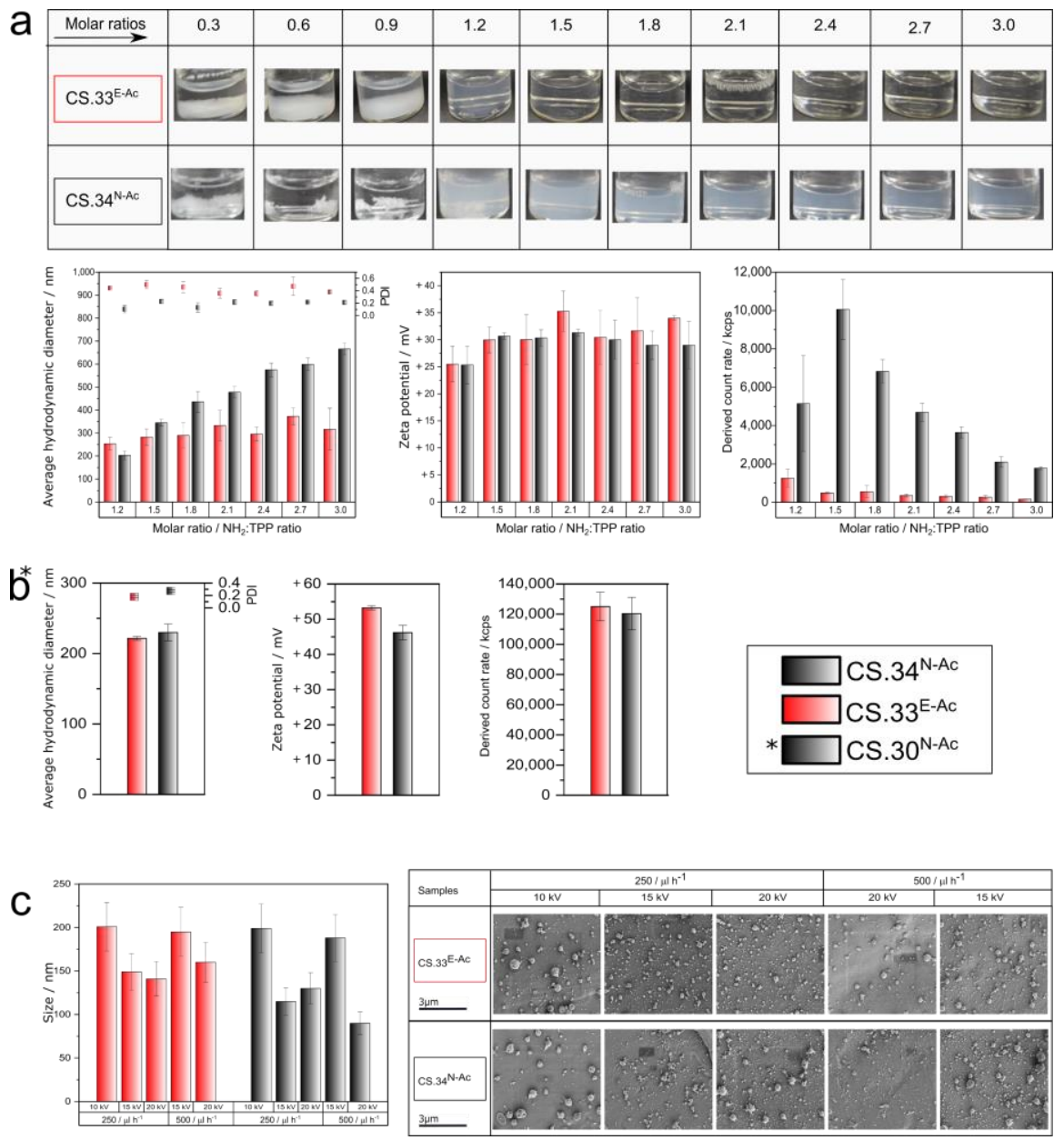

\section{Fig. 2: PA influences the nanoformulation of chitosans}

a) Physicochemical characterization of chitosan-TPP nanoparticles prepared by ionic gelation at different $\mathrm{NH}_{2} / \mathrm{TPP}$ molar ratios: images of chitosan-TPP formulations after preparation (upper panel), Z-average hydrodynamic diameter and polydispersity index (PDI) (lower left panel), zeta potential (lower center panel), and derived count rate (lower right panel) determined by dynamic light scattering. Data represent three independent experiments plotted as means \pm SD. b) Physicochemical characterization of chitosan nanocapsules prepared by solvent displacement: average hydrodynamic diameter and PDI (left panel), zeta potential (center panel), and derived count rate (right panel) determined by dynamic light scattering. Data represent three independent experiments plotted as means \pm SD. c) Characterization of electrosprayed chitosan particles: size of particles determined from scanning electron micrographs of 50 particles each (right panel) using ImageJ software, given as means \pm SE (left panel).

The quantitative solution properties of the two chitosan polymers (Fig. 3) were compared by measuring the small deformation rheology characterized by the frequency dependence profile of the elastic $\left(G^{\prime}\right)$ and loss $\left(G^{\prime \prime}\right)$ moduli and the complex viscosity $\left(\eta^{*}\right)$. Intriguingly, the profile of the conventional random-PA chitosan was typical of random coil behavior in a dilute polymer solution ${ }^{41}$, whereas the biotech block-PA chitosan showed overall lower $G^{\prime}$ and $G^{\prime \prime}$ values but the frequency dependence of both moduli displayed a similar slope (Fig. 3a). This peculiarity was also illustrated by the frequency dependence of $\tan \delta\left(=G^{\prime \prime} / G^{\prime}\right)$ for both solutions, which showed a 
monotonic decrease for the conventional chitosan, but was essentially independent of frequency for the biotech chitosan apart from an abrupt upturn above $\sim 10 \mathrm{rad} / \mathrm{s}$, which corresponds to an anomalous downturn in $\mathrm{G}^{\prime}$ and probably arises from the onset of resonance in the measuring geometry of the rheometer at high frequency. Fig. 3 also shows the corresponding Cox-Merz superposition for both systems, representing the values of $\eta^{*}$ and the steady shear viscosity $(\eta)$ as a function of oscillation frequency and shear rate, respectively. Notably, the randomPA polymer showed almost Newtonian behavior (with $\eta^{*}$ and $\eta$ showing little dependence on frequency and shear rate, respectively) and similar values of both viscosities, thus conforming to the Cox-Merz rule ${ }^{42}$. In contrast, the values of $\eta^{*}$ and $\eta$ were almost an order of magnitude lower for the block-PA polymer and yet it displayed much greater shear thinning behavior, while still conforming to the Cox-Merz rule within experimental error. These results suggest that the conventional chitosan behaves, as expected, as a polymer random coil in the entangled regime, whereas the biotech chitosan adopts a different conformation. This difference in conformation was also indicated by circular dichroism (CD) spectroscopy, which revealed subtle but important differences in the peak bands of the two samples (Fig. 3b). Indeed, both the peak and trough CD bands of the block-PA chitosan appeared shifted by $\sim 4 \mathrm{~nm}$ to a higher $\lambda$ than the random-PA chitosan. Finally, we investigated the dependence of pyrene fluorescence $\left(I_{374} / I_{385}\right.$ ratio) on the concentration of both chitosan solutions ${ }^{43,44}$ (Fig. 3c). The block-PA chitosan had a much stronger dependency than the random-PA chitosan, thus confirming the greater hydrophobicity of the block-PA polymer. The results support our hypothesis that the biotech chitosan contains a greater proportion of hydrophobic block domains than the conventional polymer. 

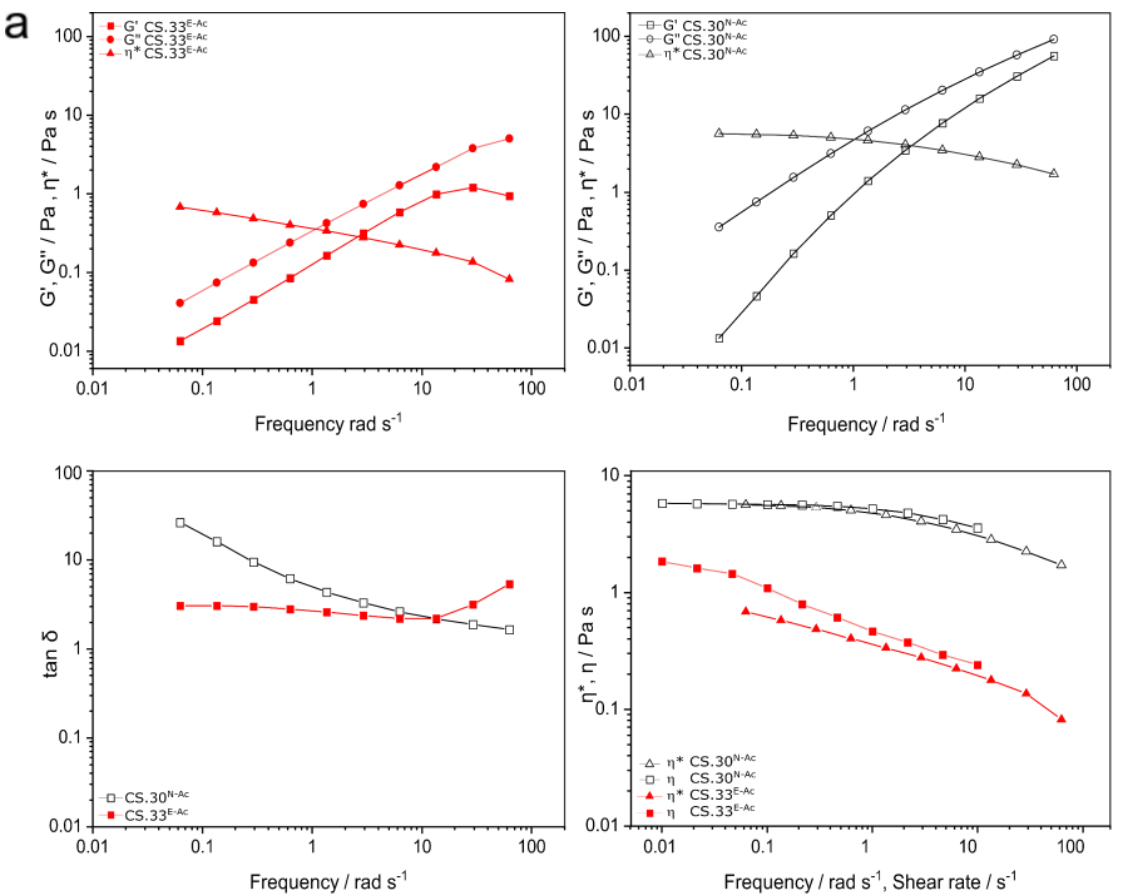

b
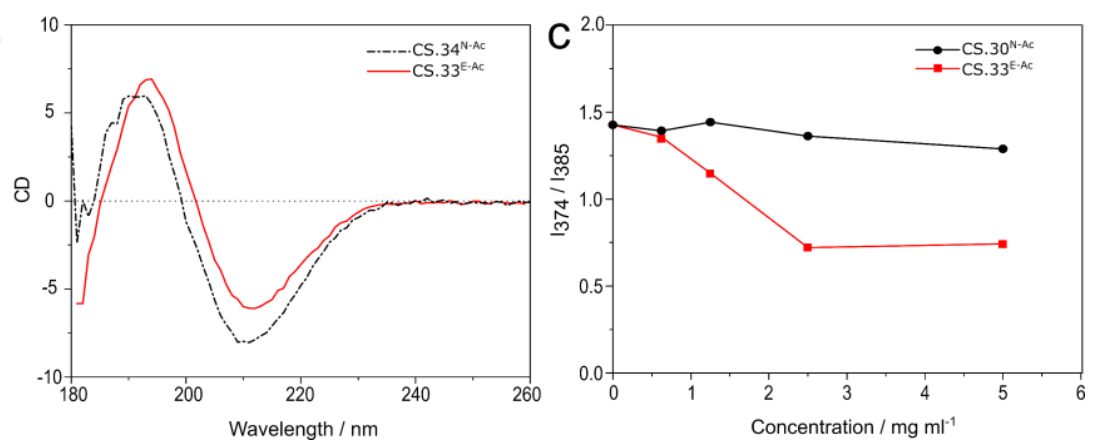

Fig. 3: The PA influences the properties of chitosans in solution

a) Rheological analysis of enzymatically (CS.33 ${ }^{\mathrm{E}-\mathrm{Ac}}$ ) and chemically (CS. $30^{\mathrm{N}-\mathrm{Ac}-1}$ ) produced chitosan polymers prepared from $30 \mathrm{mg} \mathrm{ml}^{-1}$ chitosan dissolved in a $5 \%$ stoichiometric excess of acetic acid at $25^{\circ} \mathrm{C}$ : dependence of viscoelastic moduli $\mathrm{G}^{\prime}$ and $\mathrm{G}^{\prime \prime}$ and complex viscosity $\left(\eta^{*}\right)$ on the frequency (strain $=20 \%$ ) of CS.30 ${ }^{\mathrm{E}-\mathrm{Ac}}$ (upper left panel) and CS.30 $0^{\mathrm{N}-A c-1}$ (upper right panel); dependence of $\tan \delta\left(=\mathrm{G}^{\prime \prime} / \mathrm{G}^{\prime}\right)$ on frequency (strain $=20 \%$ ) (lower left panel) and Cox-Merz representation of $\eta^{*}$ and steady-shear viscosity $(\eta)$ as a function of frequency and shear rate, respectively (lower right panel). All measurements were conducted within the linear viscoelastic region. b) Circular dichroism spectroscopy of enzymatically (CS.33 $\left.{ }^{\mathrm{E}-\mathrm{Ac}}\right)$ and chemically $\left(\mathrm{CS} .34^{\mathrm{N}-\mathrm{Ac}}\right)$ produced chitosan $(0.5 \mathrm{mg}$ $\mathrm{ml}^{-1}$ at $\left.25^{\circ} \mathrm{C}\right)$. c) Ratio of pyrene fluorescence emission intensities $\left(\mathrm{I}_{374} / \mathrm{I}_{385}\right)$ from enzymatically $\left(\mathrm{CS} .33^{\mathrm{E}-\mathrm{Ac}}\right)$ and chemically $\left(\mathrm{CS} .30^{\mathrm{N}-\mathrm{Ac}}\right)$ produced chitosan as a function of chitosan concentration $\left(\lambda_{\mathrm{ex}}=343 \mathrm{~nm}, 2 \mu \mathrm{M}\right.$ pyrene, $5 \%$ stoichiometric excess of acetic acid, $100 \mathrm{mM} \mathrm{NaCl}, 25^{\circ} \mathrm{C}$ ).

\section{The PA influences the biological activity of chitosans}

We next compared the biological activities of the two chitosan samples (Fig. 4). The enzymatically $N$-acetylated block-PA chitosan showed stronger antibacterial activity than the chemically $N$-acetylated random-PA chitosan (Fig. 4a). The antibacterial activity of conventional chitosans is inversely related to the $F_{\mathrm{A}}$. This probably reflects the decreasing charge density, because the antimicrobial activity of chitosans is thought to be caused by electrostatic interactions involving GlcN-rich blocks in the polymer chain that are large enough to disrupt membrane integrity ${ }^{45,46}$. The block-PA chitosan appears to contain sufficiently large GlcN-rich blocks to confer 
antibacterial activity even at the rather high $F_{A}$ of 0.33 , where a random-PA chitosan would have an average GlcN block size of only 3. Both the biotech and conventional chitosans showed no cytotoxicity toward HaCaT human keratinocytes (Fig. S2). The most striking difference between the two types of chitosan was their sensitivity to different chitosanolytic enzymes (Fig. 4b). Incubation of the random-PA chitosan with a bacterial chitosanase that strongly favors GIcN units close to its cleavage site ${ }^{21,47}$ predominantly yielded fully deacetylated and partially acetylated dimers and trimers. In contrast, the biotech chitosan yielded almost exclusively fully deacetylated dimers and trimers, consistent with a block-PA organization (GlcN blocks are degraded to $\mathrm{GlcN}_{2}$ and $\mathrm{GlcN}_{3}$, whereas GIcNAc blocks are not degraded). Conversely, incubation of the random-PA chitosan with a bacterial chitinase that strongly favors GIcNAc units ${ }^{19,20,48}$ predominantly yielded partially acetylated dimers and trimers but almost no fully-acetylated chitobiose. In contrast, the biotech chitosan predominantly yielded chitobiose GlcNAc2 $_{2}$ derived from the GlcNAc blocks. Similar behavior was observed using the human enzyme chitotriosidase, which has subsite preferences resembling the bacterial chitinase ${ }^{49,50}$. Human lysozyme, which has an almost absolute specificity for GIcNAc units close to its cleavage site ${ }^{51,52}$, did not produce oligomers from the randomPA substrate but generated fully-acetylated dimers to tetramers from the block-PA substrate. Similar results were obtained when the enzymatic degradation products of two chitosans with an even lower $F_{A}$ of 0.1 were compared. As anticipated given the low $F_{A}$, chitosanase generated similar products with both substrates, but chitinase and chitotriosidase yielded chitobiose alone from the block-PA chitosan. Surprisingly, lysozyme digestion of the block-PA chitosan yielded fully-acetylated GlcNAc oligomers up to DP =4, indicating the frequent presence of heptameric or even longer GlcNAc blocks in this polymer ${ }^{53}$. With a random PA, the anticipated frequency of heptameric GlcNAc blocks would be $0.1^{7}$, equivalent to one in every 10 million monomeric units, or one in every 10,000 polymers with a DP of 1000 . As expected, thus, lysozyme did not yield any oligomeric products from the random $P A$ chitosan with $F_{A}$ of $\sim 0.1$. 

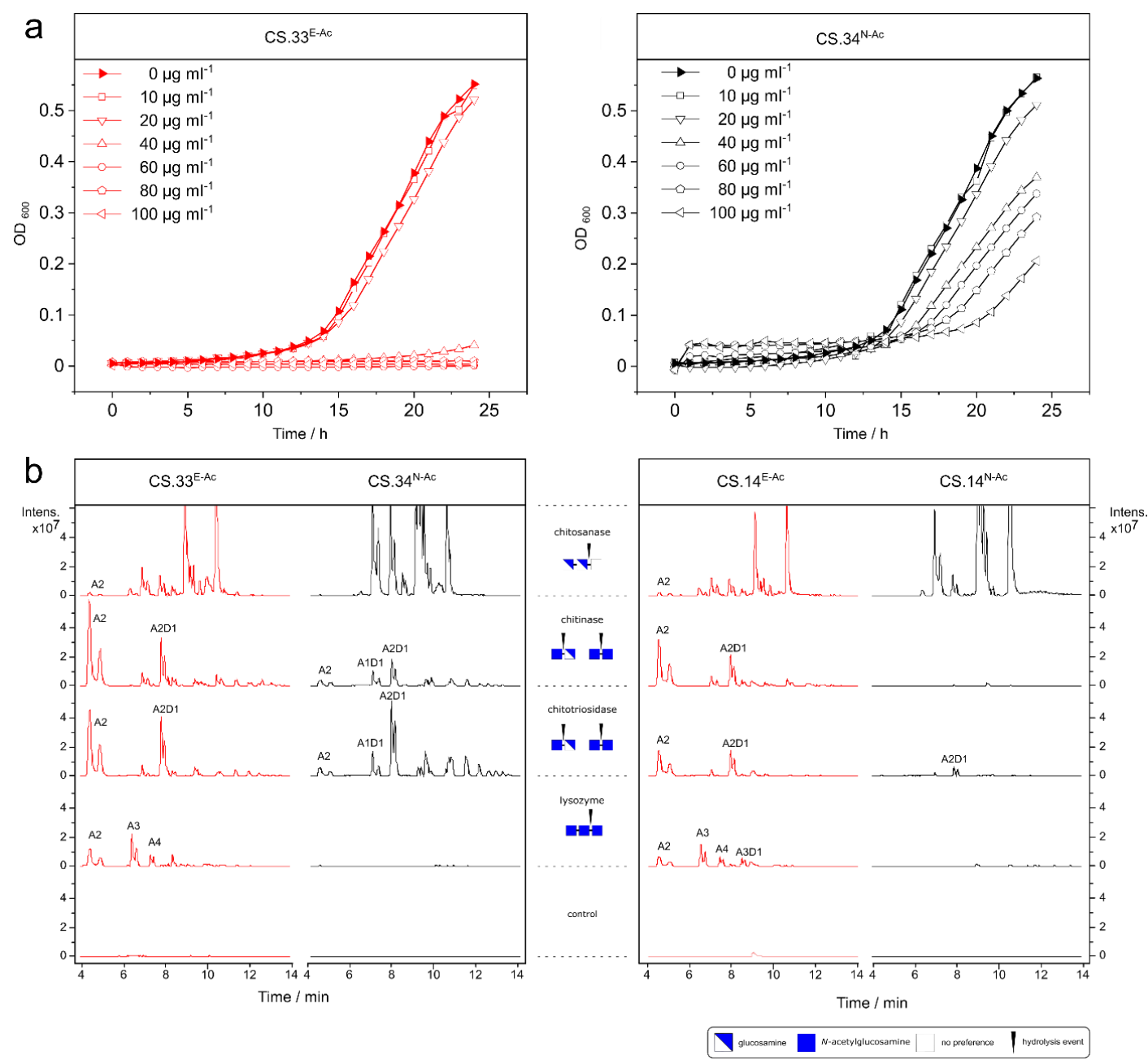

Fig. 4: The PA influences the antimicrobial activity and enzymatic degradability of chitosans

a) Growth of Pseudomonas syringae pv. tomato (DC3000 [pVSP61]) in the presence of different concentrations of enzymatically (left panel) and chemically (right panel) $N$-acetylated chitosan polymers, measured as $O D_{600}$ over $24 \mathrm{~h}$. b) UHPLC-ESI-MS analysis, showing base peak chromatograms of the oligomeric hydrolysis products ( $\mathrm{A}=\mathrm{GlcNAc}, \mathrm{D}=\mathrm{GlcN}$ ) of enzymatically (left panel) and chemically (right panel) $N$-acetylated chitosan polymers of $F_{A} \approx 0.3$ (left panel) or $F_{A} \approx 0.1$ (right panel) after $24 \mathrm{~h}$ of incubation with chitinase (ChiB), chitosanase (Csn174), human lysozyme, or human chitotriosidase, or without an enzyme. The subsite specificities of the chitinolytic enzymes are indicated in the central gutter.

While the above experiments featured chemically and enzymatically $N$-acetylated chitosans, almost all commercial chitosans are prepared by the partial de-acetylation of chitin rather than the partial $\mathrm{N}$-acetylation of polyglucosamine. We therefore selected the two enzymatically $N$-acetylated chitosans described above (the first $F_{\mathrm{A}}=0.33$ and $\mathrm{DP}=800$; the second $F_{\mathrm{A}}=0.14$ and $\mathrm{DP}=200$ ) as well as two commercial, chemically de-acetylated pharmaceutical-grade chitosans with similar parameters (the first $F_{\mathrm{A}}=0.24$ and DP $=1300$; the second $F_{\mathrm{A}}=0.17$ and DP $=200$ ) for more detailed comparative analysis (Fig. 5). The two commercial chitosans have been used in biomedical research, especially for the transfection of human cells ${ }^{54-56}$. We therefore prepared polyplexes by mixing these chitosans with plasmid DNA encoding eGFP at different molar charge chitosan/DNA ratios. For most ratios, we obtained particles with a diameter of 200-300 nm, although the high- $F_{A}$ high-DP conventional chitosan generated larger particles at higher chitosan/DNA ratios (Fig. 5a; Fig S3a). All subsequent experiments were performed at a $\mathrm{NH}_{3}{ }^{+} / \mathrm{PO}_{4}^{-}$molar charge ratio of 8 , which produced stable particles (Fig. S3b). Chitosan/DNA 
polyplexes tend to aggregate at neutral $\mathrm{pH}$ because they lose their surface charge, thus their stability in physiological media is critical for their suitability as a transfection reagent ${ }^{57}$. With the exception of the low- $F_{\mathrm{A}}$ low-DP conventional chitosan, all polyplexes were stable in OptiMEM for $4 \mathrm{~h}$ (Fig. 5b). When these polyplexes were used to transfect MCF7 breast cancer cells, only the low- $F_{A}$ low-DP biotech chitosan achieved significant transfection efficiency on par with the positive control, Lipofectamine (Fig. 5c). Importantly, both biotech chitosans were degraded by human lysozyme, as shown by the production of chitobiose and chitotriose, whereas the conventional chitosans were not (Fig. S3c). 

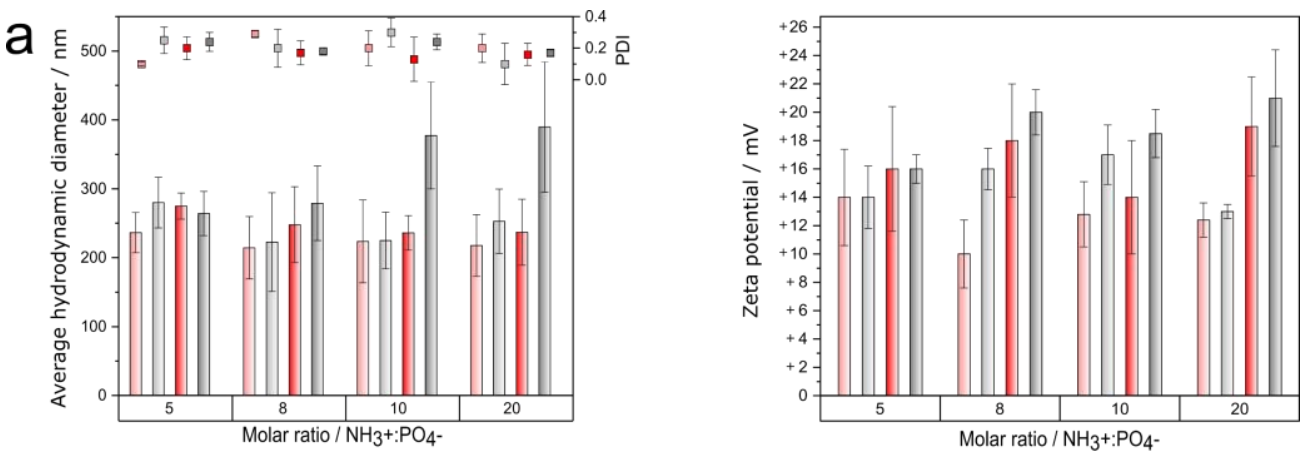

b
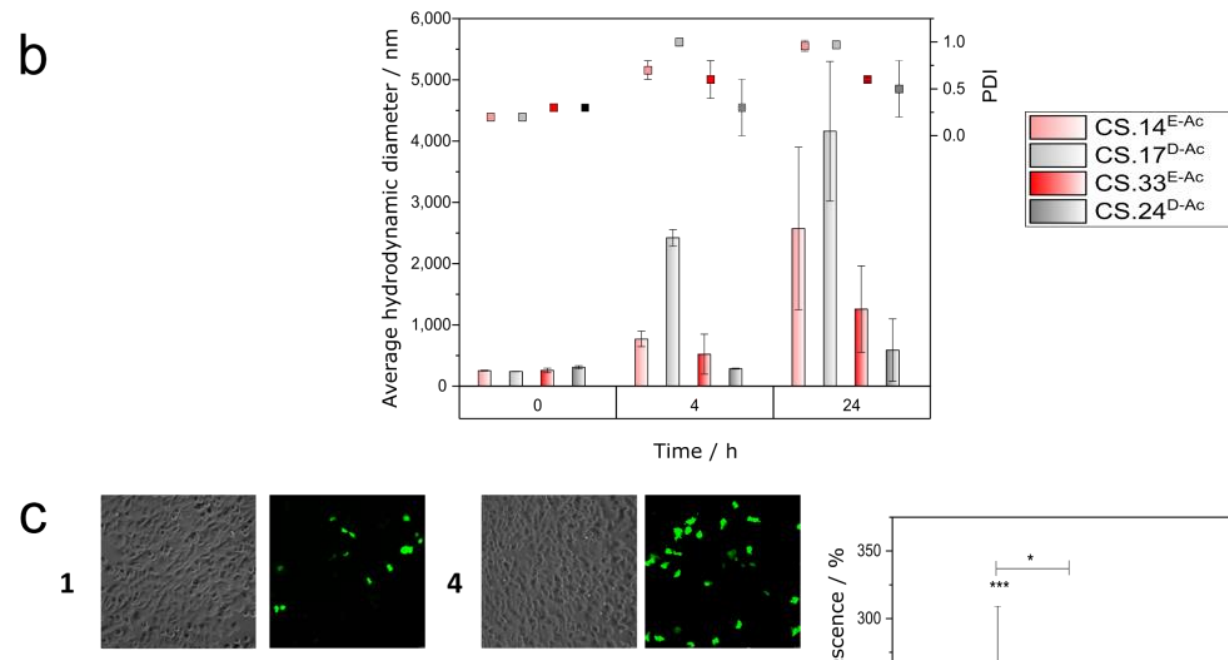

2
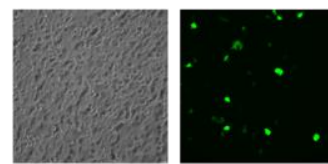

5
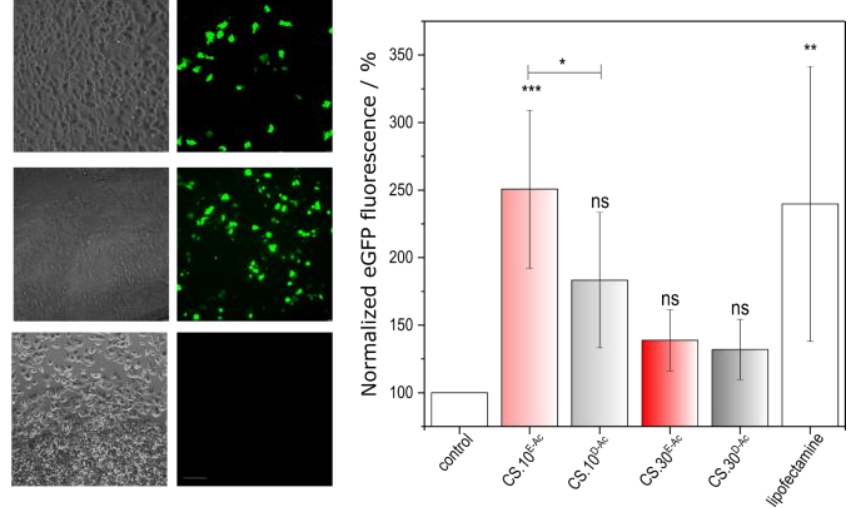

Fig. 5: The PA influences the transfection efficiency of chitosan/DNA polyplexes

a) Physicochemical characteristics of polyelectrolyte complexes formed from enzymatically $N$-acetylated and chemically de-acetylated chitosan polymers $\left(F_{\mathrm{A}} \approx 0.3\right.$ or 0.1 ) and plasmid DNA (pDNA) at different $\mathrm{NH}_{3}{ }^{+} / \mathrm{PO}_{4}^{-}$ molar charge ratios: Z-average hydrodynamic diameter and PDI (left panel) and zeta potential (right panel) as determined by dynamic light scattering. Data represent three independent experiments plotted as means \pm SD. b) Stability of chitosan/pDNA polyplexes in transfection media: the polyplexes were formed at a $\mathrm{NH}_{3}{ }^{+} / \mathrm{PO}_{4}{ }^{-} \mathrm{molar}^{-}$ charge ratio of 8 and were incubated for different times in OptiMEM at $\mathrm{pH} 6.8$ and $37^{\circ} \mathrm{C}$. Data represent three independent experiments plotted as means \pm SD. c) Transfection efficiency of the chitosan/pDNA polyplexes: Representative phase contrast and fluorescence microscopy images of MCF7 cells transfected with chitosan/pDNA polyplexes or Lipofectamine-pDNA as a positive control, showing GFP expression $48 \mathrm{~h}$ posttransfection: $1=\mathrm{CS} .33^{\mathrm{E}-\mathrm{Ac}}, 2=\mathrm{CS} .24^{\mathrm{D}-\mathrm{Ac}}, 3=\mathrm{CS} .17^{\mathrm{D}-\mathrm{Ac}}, 4=$ Lipofectamine $2000,5=\mathrm{CS} .14^{\mathrm{E}-\mathrm{Ac}}, 6=$ control cells (left panel); fluorescence intensity data normalized to negative control of MCF7 cells transfected with carriers and $2.5 \mu \mathrm{g}$ pDNA per well after $48 \mathrm{~h}$ of incubation; negative control = cells not transfected; positive control = cells transfected with Lipofectamine 2000 (right panel). Data represent three independent experiments plotted as means \pm SD. Statistical test: ANOVA followed by Tukey's multiple comparisons test $\left({ }^{*} p<0.05 ;{ }^{* *} p<0.01 ;{ }^{* * *} p<\right.$ $0.001 ; \mathrm{ns}=$ not significant compared to negative control). 


\section{Discussion}

Chitosan is a versatile biopolymer with useful biological activities, but applications have been held back by the poorly understood structure-function relationships of partially acetylated chitosans ${ }^{5,58,59}$. Recent improvements in analytical techniques and reproducible protocols for the synthesis of well-characterized 'second generation' chitosans with known DP and $F_{A}$ have provided insight into these molecules ${ }^{11,60}$. However, all commercial chitosans are produced chemically and are thought to have a random PA ${ }^{15}$, so it is not yet possible to investigate the influence of this property on chitosan behavior. We have now demonstrated that recombinant CDAs can be used to $\mathrm{N}$-acetylate polyglucosamine in the presence of excess acetate, yielding chitosan polymers with unique, non-random PAs. Two enzymatically generated chitosans featuring a block-wise distribution of acetyl groups were compared in detail to chemically generated random-PA chitosans with a similar DP and $F_{A}$. This revealed the strong influence of acetylation patterns on both the physicochemical properties and biological activity of the chitosans. The availability of these closer-to-nature 'third generation' biotech chitosans opens a new dimension in chitosan research and exploitation by allowing the control of all three key parameters.

Chitosans are binary copolymers of hydrophobic and hydrophilic subunits. In conventional chitosans, these monomeric units appear to be randomly distributed and the molecules therefore follow a general rule of behavior in aqueous solution ${ }^{61}$. At low $F_{A}(\leq 0.25)$, they show polyelectrolyte behavior, whereas at higher $F_{A}(\geq$ $0.5)$, they behave as typical hydrophobic polymers. At intermediate values, their surprisingly stable behavior is governed by the balance between hydrophilic and hydrophobic interactions. Enzymatic $N$-acetylation provides access to biotech chitosans with non-random acetylation patterns that clearly behave in a different manner to conventional chitosans in solution. For example, block-PA chitosans possess the properties of both polyelectrolytes and hydrophobic polymers in a single molecule, potentially leading to phenomena such as microphase separation as reported for other biopolymers. For example, the solution properties (such as gelling potential) of partially methylesterified homogalacturonan pectins strongly depend on both the degree and pattern of methylesterification ${ }^{62-64}$. The distribution of hydrophobic versus charged domains within these polymers governs their interactions with other, similarly heterogeneous but oppositely charged polymers. In the future, polyelectrolyte complexes of chitosans and pectins with defined patterns of acetylation and methylesterification, respectively, could facilitate the targeted, sustained, and controlled release of hydrophobic and hydrophilic drugs as well as vaccines and genes.

The biological functions of copolymers and their oligomeric degradation products are strongly influenced by their degree and pattern of substitution, as reported for pectin methylesterification ${ }^{65}$ and glycosaminoglycan sulfation ${ }^{66}$. Regarding chitosans, a crucial influence of the $F_{A}$ on biological activities has been proven for both polymers and oligomers 5,6,8,10,18,58,59, while a role of the PA was only recently revealed for chitosan oligomers $22,23,31,67$, and is reported here for the first time for chitosan polymers. In part, this reflects direct physicochemical interactions with target molecules or structures. For example, block-PA chitosans may combine the antimicrobial activity of low- $F_{A}$ chitosans ${ }^{5,45}$ with the ability of high- $F_{A}$ chitosans to induce pathogen resistance in plants 5,8 , allowing the development of dual-function plant protection products. The pattern of substitution also influences the interactions with enzymes, particularly with depolymerases such as chitinases and chitosanases 21,27, polygalacturonases and pectin/pectate lyases ${ }^{68}$, or heparinases and heparan sulfate lyases ${ }^{69,70}$. We have shown that the PA strongly influences the sensitivity of chitosan polymers to different enzymes, affecting the kinetics, nature, and quantities of the resulting oligomeric products. This is important because chitosan polymers are typically degraded in target tissues by sequence-dependent chitosan hydrolases, potentially giving rise to biologically active chitosan oligomers with a specific architecture ${ }^{31,50,67,71}$. Biodegradability is also a prerequisite for the biocompatibility of chitosans used as pharmacological excipients. Chitosan nanoparticles and nanocapsules are ideally suited for the transmembrane delivery of drugs, vaccines, and genes, ${ }^{38,72}$ but the inability of human chitinases to break down the most efficient, low- $F_{A}$ chitosans precludes their application in medicine ${ }^{50,71}$. Enzymatically $N$-acetylated low- $F_{A}$ block-PA chitosans could offer a game-changing innovation in this scenario. 
Depending on the enzyme used for $\mathrm{N}$-acetylation, chitosans can be generated with either more block-wise acetyl groups or more regular distribution of GIcNAc. Like chitinases and chitosanases ${ }^{21}$, chitosan deacetylases feature a substrate-binding cleft consisting of several subsites, each binding a single monomeric subunit ${ }^{26}$ and probably showing unique preferences or specificities for binding GIcN or GlcNAc units. The fungal enzymes used in this study are each likely to possess four subsites, ranging from $\{-2\}$ to $\{+1\}$, with subsite $\{0\}$ binding the GIcNAc unit that is deacetylated to form GIcN. Most CDAs prefer GIcNAc at all subsites, but CnCDA4 prefers GlcN at subsite $\{-1\}$ and is therefore classed as a chitosan deacetylase rather than a chitin deacetylase ${ }^{67}$. A typical chitin deacetylase, such as PesCDA or AnCDA, prefers GIcNAc at subsite $\{-1\}$ and tends to add acetyl residues downstream (further towards the reducing end) of existing GIcNAc units, thus creating GlcNAc blocks with interspersed GIcN blocks and favoring a block-PA architecture. In contrast, the chitosan deacetylase CnCDA4 tends to add acetyl groups downstream of a GlcN unit, generating chitosans with more regularly distributed GIcNAc units. PgtCDA appears to have only a slight preference for GIcN at subsite $\{-1\}^{32}$, probably explaining why it yields a chitosan with an almost random PA. The varying preferences at subsites $\{-2\}$ and $\{+1\}$ also influence the distribution of acetyl groups on the product, so that each enzyme produces chitosans with a unique PA. In this study, we investigated four closely related fungal CDAs, and the products differed for each enzyme. Given the huge diversity of naturally occurring CDAs (not only in fungi, but also in bacteria, insects, and even viruses) and the possibilities offered by protein engineering to modify subsite specificities or preferences, or alter processivity, the enzymatic $\mathrm{N}$-acetylation of polyglucosamine will allow the unlimited production of 'third generation' chitosans for research and development. Given that chitosans are the only polycationic counterparts to many different polyanionic biopolymers, the impact of this innovation will extend far beyond chitosan research into many aspects of advanced functional materials, medicine, agriculture, and industrial biotechnology.

\section{Conclusion}

In conclusion, we here report on the biotechnological production and characterization of chitosan polymers with non-random acetylation patterns, using recombinant chitin deacetylases acting in reverse mode on polyglucosamines, and their potential as advanced functional biomaterials with novel properties. In contrast to all conventional chemically produced chitosans which possess random PA, the PA of the biotech chitosans ranges from large block-like structures to near even distribution, depending on the enzyme used. These novel chitosans which differ from their conventional counterparts in terms of physicochemical properties and biological activities could become the 'third generation' of chitosans, structurally controlled in all three key parameters. Perhaps most importantly, we describe a chitosan polymer with a block-like distribution of acetyl groups that is easily degraded by human chitinases and even lysozyme, despite its low FA. Biodegradability in humans will facilitate the use of chitosan nanostructures for the delivery of drugs, genes, and vaccines, which is not possible with current chitosans. We show that this block-PA chitosan can form stable polyelectrolyte nanocomplexes with nucleic acids, achieving better transfection efficiency than conventional chitosans. These offer a game changing potential in many fields, including the development of reliable and stable RNA-based vaccines.

\section{Materials and Methods}

\section{Production and purification of enzymes \\ Chitosan Hydrolases}

The hydrolytic enzymes required for EMS fingerprinting chitinase ChiB (from Serratia marcescens, Uniprot acc. no. A0A059UJTO ${ }^{73}$ ) and chitosanase Csn174 (from Streptomyces sp. N174, Uniprot acc. no. P33665 ${ }^{47}$ ) were produced heterologously in E. coli as fusion proteins with Strep-tag II and purified using Streptactin affinity chromatography. Chitinosanase (from Alternaria alternate) was purified from the spent medium of the fungus using cation exchange chromatography, as described ${ }^{74}$. 
The chitosanolytic enzymes used to assess enzymatic digestibility of chitosan polymers were either produced heterologously in E. coli and purified as described above (chitinase ChiB; chitosanase Csn174) or expressed homologously in HEK293 cells (chitotriosidase ChT, Uniprot acc. no. Q13231 ${ }^{67}$ ) as fusion protein with His6-tag, and purified using Ni-NTA affinity chromatography.

\section{Chitin Deacetylases}

Fungal chitin deacetylases were produced recombinantly in E. coli and purified using Streptactin affinity chromatography. PesCDA (from Pestalotiopsis spec., GenBank acc. no. KY024221 31), PgtCDA (from Puccinia graminis f.sp. triciti, NCBI acc. no. XP_003323413.1 ${ }^{32}$ ), CnCDA4 (from Cryptococcus neoformans, Uniprot acc. no. Q96TR5 ${ }^{33}$ ) were produced as previously described. AnCDA was heterologously expressed in E. coli Rosetta 2 (DE3) as a truncated version ( $\Delta 1-19$, proposed signal peptide) of the chitin deacetylase An12g04480 from A. niger CBS 513.88. A C-terminal Strep-tag II and SerAla linker (SA-WSHPQFEK) was added for protein purification.

\section{Nomenclature of Chitosans}

All chitosans used in this study are named as CS.xx ${ }^{Y-A c}$, where .xx represents the $F_{A}$ of the chitosan and the superscript denotes the type of $F_{A}$-modification applied, with $\mathrm{D}=$ chemical de-acetylation, $\mathrm{N}=$ chemical $N$ acetylation, and $\mathrm{E}=$ enzymatic $N$-acetylation.

\section{Preparation of Chitosans}

\section{Chemical De-Acetylation}

Chemically de-acetylated chitosans were obtained commercially from Heppe Medical Chitosan GmbH (HMC ${ }^{+}$, Halle/Saale, Germany), namely ultrapure biomedical grade HMC 70/5 (Batch No. 212-170614-01) and HMC 70/100 (Batch No. 212-170114-01). They were produced from snow crab shell $\alpha$-chitin using a heterogeneous de- $N$-acetylation process in hot alkali ${ }^{75}$

\section{Chemical $\mathbf{N}$-Acetylation}

Chemically $\mathrm{N}$-acetylated chitosans were prepared using acetic anhydride, as previously described ${ }^{60}$. Briefly, chitosan (DP 1300, $€$ 1.8, $F_{\mathrm{A}}$ 0.03; prepared from shrimp shell $\alpha$-chitin by four sequential heterogeneous de- $N$ acetylation steps using hot alkali by Mahtani Chitosan, Veraval, India ${ }^{75}$ was solubilized in water by adding $5 \%$ stoichiometric excess of acetic acid and stirred until completely dissolved. One volume of 1,2-propanediol was added to the chitosan solution to reduce the isoelectric constant of the medium and to help chitosan chains to adopt an open conformation. Acetic anhydride was then added in the required molar amount to reach the target $F_{\mathrm{A}}$. After $24 \mathrm{~h}$ at RT, chitosan was precipitated with an ammonia solution ( $\left.23 \% \mathrm{w} / \mathrm{v}\right)$. The polymer precipitate was washed to neutrality and freeze-dried for subsequent use.

\section{Enzymatic $\mathbf{N}$-Acetylation}

Enzymatically $N$-acetylated chitosans were produced using different fungal chitin deacetylases recombinantly produced in $E$. coli as described above. The same chitosan polymer as described above for chemical $\mathrm{N}$-acetylation was used as a starting material and dissolved in $5 \%$ stoichiometric excess of acetic acid to achieve complete dissolution. This solution was further diluted $1: 2$ in $3 \mathrm{M}$ sodium acetate buffer $\mathrm{pH} 7.5$ to a final chitosan concentration of $1 \mathrm{mg} \mathrm{mL}^{-1}$. The small-scale $(2 \mathrm{~mL})$ time series experiments were performed for $24 \mathrm{~h}$ at $37^{\circ} \mathrm{C}$ using the following concentration of enzymes: AnCDA, $1.2 \mu \mathrm{M}$; CnCDA4, 700 nM; PesCDA, 175 nM; PgtCDA, 600 $\mathrm{nM}$. Samples were taken at different time points to determine the $F_{\mathrm{A}}$ by EMS fingerprinting as described below. The large-scale $(3.5 \mathrm{~L}$ ) production using PesCDA was performed under the same conditions as described above, the $F_{\mathrm{A}}$ was monitored using EMS fingerprinting and additional enzyme was added until the desired $F_{\mathrm{A}}$ was reached. Then, chitosan was precipitated using acetone (1:1 v:v), the precipitate was collected by centrifugation (20 $\mathrm{min}, 12,000 \times \mathrm{g}, 4^{\circ} \mathrm{C}$ ), washed three times with water adjusted to $\mathrm{pH} 9$ using ammonia, and then three more times with distilled water, before being freeze dried. To ensure complete removal of salts remaining from high salt conditions required for enzymatic $N$-acetylation, samples were re-dissolved in water and dialysis was performed using 12 kDa cut-off dialysis membranes (Repligen, Ravensburg, Germany). Samples were again freeze dried before being used for structural and functional analysis. 


\section{Structural Analysis of Chitosans}

Chitosan polymers were structurally analyzed using well-established, previously described methods. The values obtained for all chitosans used in this study are given in Supplementary Table S2.

\section{Degree of Polymerization}

The number and weight average DP of chitosan polymers were determined based on previous work with slight

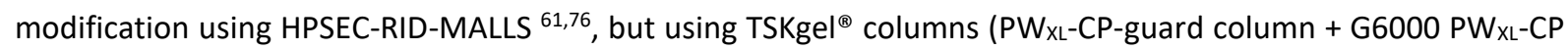
+ G5000 PWXL-CP + G3000 PWXL-CP) (Tosoh, Griesheim, Germany) and degassed ammonium acetate buffer ( 0.15 $\mathrm{M}, \mathrm{pH}$ 4.5) as an eluent at a flow rate of $0.5 \mathrm{~mL} \mathrm{~min}^{-1}$. Light scattering intensity measurements were performed to determine the $\mathrm{Mw}$ and $\mathrm{Mn}$ following the Rayleigh-Debye equation (using WinGPC UniChrom sofwater, PSS, Germany), consequently these values were used to calculate $\bigoplus$.

\section{Fraction of Acetylation}

The average $F_{A}$ of chitosan polymers was determined either using ${ }^{1} \mathrm{H}-\mathrm{NMR}{ }^{77}$ recording $200-250$ spectra on a AV300 or DPX300 300 MHz spectrometer (Bruker,USA), or using chitinase/chitosanase-mass spectrometric fingerprinting ${ }^{78}$.

\section{Pattern of Acetylation}

The PA of chitosan polymers was determined either using ${ }^{13} \mathrm{C}-\mathrm{NMR}$ dyad analysis ${ }^{15}$ or using chitinosanase-mass spectrometric fingerprinting ${ }^{27,76}$. For ${ }^{13} \mathrm{C}-\mathrm{NMR}$ analysis, $100 \mathrm{mg}$ of purified chitosan was dissolved in $10 \mathrm{~mL}$ of $0.07 \mathrm{M} \mathrm{HCl}$ and stirred overnight at room temperature. The solution was treated with $10 \mathrm{mg}$ sodium nitrite $\left(\mathrm{NaNO}_{2}\right)$ for partial depolymerization, stirred for $4 \mathrm{~h}$, and subsequently freeze dried. Samples were dissolved in $1 \mathrm{~mL}$ acidic solution of $\mathrm{D}_{2} \mathrm{O}\left(1 \mathrm{ml} 99.9 \% \mathrm{D}_{2} \mathrm{O}, 5 \mu \mathrm{LCl}\right)$ and freeze-dried. Finally, samples were dissolved in $\mathrm{D}_{2} \mathrm{O}$, and ${ }^{13} \mathrm{C}$-NMR spectra were recorded on a $600 \mathrm{MHz}$ DD2 instrument (Agilent, USA). The dyad frequencies of chitosan samples were determined based on the $\mathrm{C}-5$ resonance region, and deviation from random statistics $\left(P_{\Sigma}\right)$ was analyzed as recently described ${ }^{27}$ based on former work by Varum et al. and Weinhold et al. ${ }^{15,16,79,80}$. For chitinosanase-mass spectrometric fingerprinting ${ }^{76}$, chitosan samples were hydrolyzed with purified chitinosanase ${ }^{74}$ (see above) using the following conditions: $1 \mathrm{mg} \mathrm{mL}^{-1}$ chitosan, $3.5 \mu \mathrm{g} \mathrm{mL}^{-1}$ chitinosanase, 200 $\mathrm{mM}$ ammonium acetate buffer, $\mathrm{pH} 4.2$, incubation at $37^{\circ} \mathrm{C}$ for two days. After one day of incubation, chitinosanase concentration was increased to $6.8 \mu \mathrm{g} \mathrm{mL}^{-1}$. Semi-quantitative HILIC-ESI-MS measurements were performed as previously described ${ }^{74,76}$. Normalized mass-fractions of the hydrolysis products with DP 2-10 were calculated. Based on the DA/XX cleavage preference of the chitinosanase, we calculated the frequencies of block sizes represented by the detected hydrolysis products. Weight-average block sizes and differences of block size frequencies between chemically and enzymatically acetylated chitosans were calculated as well.

\section{Physico-Chemical Solution Properties of Chitosans}

\section{Microviscosimetry}

The dynamic viscosity of chitosan aqueous solutions, solubilized with $5 \%$ stoichiometric excess of acetic acid and $0.1 \mathrm{M} \mathrm{NaCl}$ was measured using an AMVn automated rolling ball microviscometer (Anton Paar, Ostfildern, Germany), using a capillary of $1.6-\mathrm{mm}$ diameter at an angle of $40^{\circ}$ and at $25^{\circ} \mathrm{C}$. The dynamic viscosity was calculated from the average of four runs, either in water containing $5 \%$ stoichiometric excess of acetic acid or in $0.1 \mathrm{M} \mathrm{NaCl}$. From the relative viscosity $\eta_{\text {rel }}$ thus determined, the specific viscosity $\eta_{s p}\left(\eta_{s p}=\eta_{\text {rel }}-1\right)$ was calculated by joint extrapolation to "zero concentration" of the Huggins, Kraemer and "single point" relationships ${ }^{81}$

\section{Nanoformulation}

Chitosan-tripolyphosphate (TPP) nanoparticles were prepared using the ionic gelation process described by Calvo et al. ${ }^{36}$ with slight modifications ${ }^{37}$. A series of $\mathrm{NH}_{2} / \mathrm{TPP}$ molar ratios $(0.3,0.6,0.9,1.2,1.5,1.8,2.1,2.4,2.7$, and 3 ) were screened to test the formation of particles. For this, stock solutions of chitosans and TPP were prepared at $2 \mathrm{mg} \mathrm{mL}^{-1}$ (filtered using $0.45 \mu \mathrm{m}$ filter) and $7 \mathrm{mg} \mathrm{mL}^{-1}$ (filtered using $0.22 \mu \mathrm{m}$ filter), respectively. ChitosanTPP particles were generated spontaneously upon dropwise addition of TPP into the chitosan solution stirring at $750 \mathrm{rpm}$ and at room temperature. All particles were prepared at a chitosan:TPP volume ratio of 3:1. The resulting particles were characterized for their size, polydispersity index (PDI), and derived count rate (DCR) using dynamic light scattering with non-invasive back scattering (DLS-NIBS) at a measurement angle of $173^{\circ}$ using the method of cumulants. The zeta potential was measured by mixed laser Doppler velocimetry and phase analysis 
light scattering (M3-PALS). A Malvern Zetasizer NanoZS (Malvern Panalytical, Malvern, UK) fitted with a red laser $(\lambda=632.8 \mathrm{~nm}$ ) was used for both analyses. The Zeta Sizer Software (v 7.12, Malvern Panalytical) was used to acquire and evaluate the data.

Nanocapsules were prepared by the solvent displacement technique first described by Calvo et al. ${ }^{82}$, with some modifications. Briefly, an organic phase was formed by dissolving $40 \mathrm{mg}$ of lecithin (Epikuron 145V, Cargill Deutschland $\mathrm{GmbH} \&$ Co. KG, Hamburg, Germany) in $1 \mathrm{~mL}$ of ethanol, followed by the addition of $125 \mu \mathrm{L}$ of Miglyol $^{\circledR} 812$ (Sasol $\mathrm{GmbH}$, Witten, Germany) and adding ethanol up to $10 \mathrm{~mL}$. This organic phase was immediately poured over $20 \mathrm{~mL}$ of the aqueous phase composed of a chitosan solution $\left(0.5 \mathrm{mg} \mathrm{mL}^{-1}\right.$ dissolved in water with $5 \%$ stoichiometric excess of acetic acid). Nanocapsules were formed spontaneously due to the organic solvent's diffusion and Marangoni effects of the organic phase ${ }^{83}$. Finally, the ethanol and some of the water were evaporated at $40^{\circ} \mathrm{C}$ under vacuum on a R-210 Rotavapor (Büchi Labortechnik, Essen, Germany) and the volume of the formulations was reduced to $10 \mathrm{~mL}$. Nanocapsules were characterized on the basis of average size distribution, PDI, DCR, and zeta potential, as described above.

To prepare chitosan nanoparticles by electrospraying, chitosans were dissolved in $30 \%$ acetic acid and $30 \%$ ethanol at a concentration of $5 \mathrm{mg} \mathrm{mL}^{-1}$ as described previously ${ }^{40}$. For the preparation of the solutions, chitosan samples were first dispersed in water, followed by the addition of acetic acid and ethanol. Mixtures were stirred overnight prior to the electrospraying process. Chitosan solutions were electrosprayed using a high voltage generator (ES50P-10W, Gamma High Voltage Research, Ormond Beach, FL, USA) and a syringe pump (New Era Pump Systems, Farmingdale, NJ, USA) to provide specific voltages and solution flow rates, respectively. For scanning electron microscopy (SEM) to investigate the morphology of the particles, the samples were attached on metal stubs with double-sided adhesive carbon tape and coated with $6 \mathrm{~nm}$ of gold for better conductivity using a sputter coater (Leica Coater ACE 200, Leica, Vienna, Austria), prior to visualization using a Quanta FEG 3D SEM (FEI, Eindhoven, The Netherlands). The average particle size (50 particles per image) was determined using ImageJ (version 1.5).

\section{Rheology}

The rheological properties of chitosans were measured at a concentration of $30 \mathrm{mg} \mathrm{mL}^{-1}$ (dissolved in $5 \%$ stoichiometric excess of acetic acid) using a Kinexus Ultra rheometer (Malvern Panalytical, Malvern, UK). A cone plate model (CP 4/40, PL 65) was used to monitor the storage and loss modulus. Frequency sweep measurements were performed where the frequency varied between 0.01 and $100 \mathrm{~Hz}\left(0.0628-62.8 \mathrm{rad} \mathrm{s}^{-1}\right)$ and the strain was $20 \%$ (within the linear viscoelastic region). Shear viscosity studies were performed at a shear rate of $0.01-10 \mathrm{~s}^{-1}$. All samples were measured at $25^{\circ} \mathrm{C}$. As the rheological measurements required relatively high amounts of chitosan which were not available for the chemically $N$-acetylated chitosan of $F_{A} 0.30$ and DP 700 that was used for all other experiments, a chitosan of $F_{A} 0.30$ and DP 1700 was used for the rheology experiments. Even when the DP does influence the overall magnitude of the viscoelastic and steady shear viscosity parameters, the comparison between the two polymers of varying DP is valid as long as both solutions are in the entangled regime. ${ }^{37,41}$

\section{Circular Dichroism}

Circular Dichroism (CD) spectroscopy was performed using a Chirascan Plus CD spectrophotometer (Applied Photophysics, Surrey, UK) with a LAAPD detector and Chirascan Spectrometer Control Panel software version 4.4 (Applied Photophysics). Far-UV CD analysis was performed from 180 to $260 \mathrm{~nm}$ with a $0.5 \mathrm{~nm}$ step size. Chitosan samples were dissolved in stoichiometric excess of acetic acid at concentrations of $0.5 \mathrm{mg} / \mathrm{mL}$. All measurements were performed at $25^{\circ} \mathrm{C}$ using a $0.1 \mathrm{~mm}$ precision cuvette (Hellma, Müllheim, Germany); each sample was scanned ten times and results were averaged, no smoothing was used. The sample solvent was also scanned under identical conditions and subtracted from the sample spectra.

\section{Pyrene Binding}

Pyrene was selected as a hydrophobic fluorescent probe to determine the hydrophobic domains within the chitosan samples. Pyrene dissolved in methanol was added to chitosan solutions $(0.625,1.25,2.5$, and $5.0 \mathrm{mg}$ $\mathrm{mL}^{-1}$ in $100 \mathrm{mM} \mathrm{NaCl}$ ) to give a final concentration of $2 \mu \mathrm{M}$. Fluorescence spectra were taken on a Jasco FP-6500 spectrofluorometer (Jasco, Pfungstadt, Germany) at $25^{\circ} \mathrm{C}$. The excitation wavelength was fixed to $343 \mathrm{~nm}$, and emission spectra were recorded between 360 and $550 \mathrm{~nm}$; the ratio between the peak intensities of the first 
peak at $374 \mathrm{~nm}\left(/ /_{1}\right)$ and of the third peak at $385 \mathrm{~nm}(/ 3)$, which vary based on the hydrophobicity of the sample, was plotted ${ }^{84,85}$.

\section{Biological Functionalities of Chitosans}

\section{Antibacterial activity}

In vitro antibacterial activity of chitosans was tested against Pseudomonas syringae pv. tomato (DC3000 [pVSP61]) provided by MPI, Cologne, Germany. Bacteria were initially grown in NYG medium $(0.5 \%(\mathrm{w} / \mathrm{v})$ peptone, $0.3 \%(\mathrm{w} / \mathrm{v})$ yeast extract, $2 \%(\mathrm{v} / \mathrm{v})$ glycerol) at $30^{\circ} \mathrm{C}$ under agitation at $100 \mathrm{rpm}$ for two days. Antibacterial assay was performed in a 96 -well plate by mixing $40 \mu \mathrm{L}$ of chitosan to $160 \mu \mathrm{L}$ of bacterial suspension with $\mathrm{OD}=0.0125$ or medium as a blank. Growth of bacteria was measured continuously for $24 \mathrm{~h}$ at an interval of $10 \mathrm{~min}$, by measuring the optical density at $\lambda=600 \mathrm{~nm}\left(\mathrm{OD}_{600}\right)$ using a UV/Vis microplate reader (SpectraMax $\mathrm{M} 2$, Molecular Devices, Sunnyvale, CA, USA) at $26^{\circ} \mathrm{C}$.

\section{Cytotoxicity}

The in vitro cytotoxicity of chitosans was studied using the MTT assay on HaCat cells as a model cell line (obtained from the dermatological clinic at the University Hospital in Münster, Germany). A HaCaT cell suspension (100 $\mu$ l containing ca. $10^{4}$ cells per well) was seeded into a 96-well tissue culture plate and incubated for $24 \mathrm{~h}$, cells were then washed twice with PBS before addition of samples at varying concentrations and further incubation for 24 h. Samples were then removed and replaced by $100 \mu$ medium containing $25 \mu \mathrm{l}$ of MTT solution $\left(5 \mathrm{mg} \mathrm{mL}^{-1}\right.$ in PBS). Following incubation for $3 \mathrm{~h}$, the medium was replaced by $100 \mu \mathrm{l}$ of DMSO and the plate was shaken for 10 $\mathrm{min}$ at $300 \mathrm{rpm}$. Absorbance was measured at $\lambda=570 \mathrm{~nm}$ using a UV/Vis microplate reader (Multiscan GO 60, Thermo Fisher Scientific, Waltham, MA, USA). Relative viability was calculated in percent of the OD value of cells growing in the absence of chitosan. Triton X-100 (Sigma Aldrich, St. Louis, MO, USA) in PBS (4\%) was used as a positive control.

\section{Enzymatic Degradation}

In vitro degradation of chitosans was performed using four different enzymes - chitinase chiB, chitosanase Csn174, egg white lysozyme (Roth, Germany), and human chitotriosidase ChT- sourced as described above. Chitosans were dissolved at $1 \mathrm{mg} \mathrm{mL}^{-1}$ in $150 \mathrm{mM}$ ammonium acetate buffer $\mathrm{pH} 4.2$ and incubated for $24 \mathrm{~h}$ at $37^{\circ} \mathrm{C}$ with $30 \mu \mathrm{g} \mathrm{mL} \mathrm{L}^{-1}$ of enzyme, or without enzyme as a control. Degradation products were analyzed using UHPLC-ESI-MS ${ }^{n}$ (Dionex Ultimate 3000RS UHPLC; Thermo Scientific, Milford, USA) via an Acquity UHPLC BEH Amide column $(1.7 \mu \mathrm{M}, 2.1 \times 150 \mathrm{~mm})$ in combination with a VanGuard precolumn $(1.7 \mu \mathrm{M}, 2.1 \times 5 \mathrm{~mm})$, both from Waters Corporation (Milford, USA), coupled to an ESI-MS detector (amaZon speed, Bruker Daltonics, Bremen, Germany). Eluent A consisted of $80 \%(\mathrm{v} / \mathrm{v})$ acetonitrile, and eluent B consisted of $20 \%(\mathrm{v} / \mathrm{v})$ acetonitrile, both supplemented with $10 \mathrm{mM} \mathrm{NH}_{4} \mathrm{HCO}_{2}$ and $0.1 \%(\mathrm{v} / \mathrm{v})$ formic acid. A column oven temperature of $35^{\circ} \mathrm{C}$ was used, and mass spectra were determined in a positive mode over scan range of $\mathrm{m} / \mathrm{z} 50-2000$. The parameters for the electrospray ionization were capillary voltage $4 \mathrm{kV}$, end plate offset voltage $500 \mathrm{~V}$, nebulizer pressure 1 bar, flow rate of the dry gas $8 \mathrm{~L} \mathrm{~min}^{-1}$, and dry temperature $200^{\circ} \mathrm{C}$. Mass spectra were analyzed using Data Analysis 4.1 software (Bruker Daltonics, Bremen, Germany). To investigate the hydrolysis of the polymers, $2 \mu \mathrm{l}$

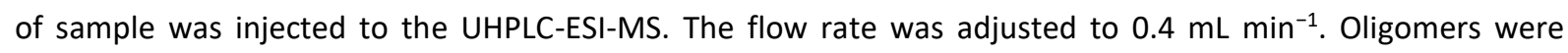
separated over a 16 min gradient elution profile: 0-2.5 min isocratic $100 \%$ A (80:20 ACN: $\mathrm{H}_{2} \mathrm{O}$ with $10 \mathrm{mM}$ $\mathrm{NH}_{4} \mathrm{HCO}_{2}$ and $\left.0.1 \%(\mathrm{v} / \mathrm{v}) \mathrm{HCOOH}\right) ; 2.5-12.5$ min linear from $0 \%$ to $75 \% \mathrm{~B}\left(20: 80 \mathrm{ACN}: \mathrm{H}_{2} \mathrm{O}\right.$ with $10 \mathrm{mM} \mathrm{NH}_{4} \mathrm{HCO}_{2}$ and $0.1 \%(\mathrm{v} / \mathrm{v}) \mathrm{HCOOH}$ ); column re-equilibration: $12.5-13.5$ min linear from $75 \% \mathrm{~B}$ to $100 \% \mathrm{~A} ; 13.5-16 \mathrm{~min}$ isocratic $100 \% \mathrm{~A}$.

\section{Transfection of human cells}

To assess the transfection efficiency of chitosan/DNA polyplexes, the human breast cancer cell line MCF7 was used (Hölzel Diagnostika GmbH, Germany). Plasmid DNA NTC8685-eGFP (3818 bp) was purchased from Nature Technology Corporation (Lincoln, NE, USA), multiplied in E. coli $\mathrm{DH} 5 \alpha$, and purified using the kit according to the manufacturer's instruction. Purity was confirmed by $1 \%$ agarose gel electrophoresis, and DNA concentration was measured by Nanodrop. For chitosan/pDNA polyplex preparation, $10 \mu \mathrm{L}$ of plasmid DNA with a concentration of $0.25 \mu \mathrm{g} \mathrm{L}^{-1}$ were mixed with $10 \mu \mathrm{L}$ of chitosan solution previously diluted in $0.1 \mathrm{M}$ of MES buffer to yield the desired $\mathrm{NH}_{3}{ }^{+} / \mathrm{PO}_{4}{ }^{-}$molar charge ratios of $5,8,10$, or 20 . After addition of $35 \mu \mathrm{L}$ of $\mathrm{MES}$ buffer $(0.1 \mathrm{M}, \mathrm{pH} 5.8)$, samples were vortexed thoroughly and incubated for $30 \mathrm{~min}$ at room temperature to allow for self-assembly of 
the polyplexes. Polyplexes were characterized in terms of size and zeta potential as described above. To assess the stability in the physiological medium used for the transfection assay, $50 \mu \mathrm{L}$ of freshly prepared polyplexes

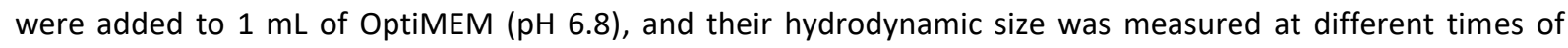
incubation at $37^{\circ} \mathrm{C}$ as described above. The binding strength between chitosan and pDNA in the polyplexes was evaluated in a gel retardation assay for which $500 \mathrm{ng}$ of free pDNA and chitosan/pDNA polyplexes in $20 \mu \mathrm{L}$ TAE buffer supplemented with $3 \mu \mathrm{L}$ of PicoGreen were loaded onto a $1 \%$ agarose gel. The gel was run at $140 \mathrm{~V}$ for 45 min; DNA bands were visualized using UV illumination.

For the in vitro transfection assay, MCF-7 cells ( 100.000 cells/well) were seeded in a 24 well plate using $1 \mathrm{~mL} /$ well of RPMI medium containing $10 \%$ FBS, $1 \%$ penicillin/streptomycin, and $1 \%$ L-glutamine. The cells were left to attach overnight at $37^{\circ} \mathrm{C}$ and $5 \% \mathrm{CO}_{2}$. Polyplexes were prepared $30 \mathrm{~min}$ prior to incubation with cells and diluted with serum-free transfection medium (OptiMEM I, Gibco; $\mathrm{pH}$ adjusted to 6.8) to a concentration of $2.5 \mu \mathrm{g}$ pDNA $\mu \mathrm{L}^{-1}$. Lipofectamine 2000 (Invitrogen, Karlsruhe, Germany; $1 \mu \mathrm{L}$ per $2.5 \mu \mathrm{g}$ of pDNA) was used as a positive control. Cells were incubated with the polyplexes for $24 \mathrm{~h}$ at $37^{\circ} \mathrm{C}$ and $5 \% \mathrm{CO}_{2}$. Then, the transfection medium was replaced by RPMI medium and incubated for another $24 \mathrm{~h}$ for protein expression. Control cells were incubated with medium only. After 48 hours, the transfection efficiency was evaluated qualitatively by the analysis of the GFP fluorescence intensities $\left(\lambda_{\mathrm{ex}}=488 \mathrm{~nm}, \lambda_{\mathrm{em}}=509 \mathrm{~nm}\right.$ ) using a fluorescence microscope (DMi8 automated S/N 409984, Leica Microsystems CMS GmbH, Wetzlar, Germany). Images were recorded using a $10 \mathrm{X} / 0.30 \mathrm{DRY}$ objective and a digital camera (Hamamatsu Flash 4.0-USB3-002560, Japan). Fluorescence intensity was quantified with Tecan ultra Evolution (Safire, Tecan, Salzburg, Austria) in top measurement mode, with manually fixed gain at 100 and ten flashes. Biological experiments were conducted at least in triplicates and with at least three technical replicates per independent experiment. Data were analyzed using Tukey multiple comparison tests with a single pooled variance using GraphPad Software Prism v6 (San Diego, CA, USA). Differences were considered statistically significant when $\mathrm{p}<0.05\left({ }^{*}\right), \mathrm{p}<0.01(* *)$, or $\mathrm{p}<0.001\left({ }^{* *}\right)$. 


\section{References}

1. El Gueddari, N. E., Rauchhaus, U., Moerschbacher, B. M. \& Deising, H. B. Developmentally regulated conversion of surface-exposed chitin to chitosan in cell walls of plant pathogenic fungi. New Phytol. 156, 103-112 (2002).

2. Merzendorfer, $\mathrm{H}$. The cellular basis of chitin synthesis in fungi and insects: Common principles and differences. Eur. J. Cell Biol. 90, 759-769 (2011).

3. Collado-González, González Espinosa \& Goycoolea. Interaction Between Chitosan and Mucin: Fundamentals and Applications. Biomimetics 4, 32 (2019).

4. Fuenzalida, J. \& Goycoolea, F. Polysaccharide-Protein Nanoassemblies: Novel Soft Materials for Biomedical and Biotechnological Applications. Curr. Protein Pept. Sci. 16, 89-99 (2015).

5. Wattjes, J. et al. Patterns matter part 1: Chitosan polymers with non-random patterns of acetylation. React. Funct. Polym. 151, 104583 (2020).

6. Kauss, H., Jeblick, W. \& Domard, A. The degrees of polymerization and N-acetylation of chitosan determine its ability to elicit callose formation in suspension cells and protoplasts of Catharanthus roseus. Planta 178, 385-392 (1989).

7. Lamarque, G., Lucas, J.-M. M., Viton, C. \& Domard, A. Physicochemical Behavior of Homogeneous Series of Acetylated Chitosans in Aqueous Solution: Role of Various Structural Parameters. Biomacromolecules 6, 131-142 (2005).

8. Vander, P., Vårum, K. M., Domard, A., Eddine El Gueddari, N. \& Moerschbacher, B. M. Comparison of the Ability of Partially N -Acetylated Chitosans and Chitooligosaccharides to Elicit Resistance Reactions in Wheat Leaves. Plant Physiol. 118, 1353-1359 (1998).

9. Malerba, M. \& Cerana, R. Recent Advances of Chitosan Applications in Plants. Polymers (Basel). 10, 118 (2018).

10. Younes, I., Sellimi, S., Rinaudo, M., Jellouli, K. \& Nasri, M. Influence of acetylation degree and molecular weight of homogeneous chitosans on antibacterial and antifungal activities. Int. J. Food Microbiol. 185, 57-63 (2014).

11. Cord-Landwehr, S., Niehues, A., Wattjes, J. \& Moerschbacher, B. M. New Developments in the Analysis of Partially Acetylated Chitosan Polymers and Oligomers. in Chitin and Chitosan 81-95 (John Wiley \& Sons, Ltd, 2019). doi:10.1002/9781119450467.ch4

12. Aiba, S. Studies on chitosan: 3. Evidence for the presence of random and block copolymer structures in partially Nacetylated chitosans. Int. J. Biol. Macromol. 13, 40-44 (1991).

13. Ottøy, M. H., Vårum, K. M. \& Smidsrød, O. Compositional heterogeneity of heterogeneously deacetylated chitosans. Carbohydr. Polym. 29, 17-24 (1996).

14. Hirano, S., Tsuneyasu, S. \& Kondo, Y. Heterogeneous distribution of amino groups in partially N-acetylated derivatives of chitosan. Agric. Biol. Chem. 45, 1335-1339 (1981).

15. Weinhold, M. X., Sauvageau, J. C. M., Kumirska, J. \& Thöming, J. Studies on acetylation patterns of different chitosan preparations. Carbohydr. Polym. 78, 678-684 (2009).

16. Vårum, K. M., Antohonsen, M. W., Grasdalen, H. \& Smidsrød, O. Determination of the degree of N-acetylation and the distribution of $\mathrm{N}$-acetyl groups in partially N-deacetylated chitins (chitosans) by high-field n.m.r. spectroscopy. Carbohydr. Res. 211, 17-23 (1991).

17. Cord-Landwehr, S. et al. Patterns matter part 2: Chitosan oligomers with defined patterns of acetylation. React. Funct. Polym. 151, 104577 (2020).

18. Aam, B. B. et al. Production of Chitooligosaccharides and Their Potential Applications in Medicine. Mar. Drugs 8, 1482-1517 (2010).

19. Cord-Landwehr, S. et al. Quantitative Mass-Spectrometric Sequencing of Chitosan Oligomers Revealing Cleavage Sites of Chitosan Hydrolases. Anal. Chem. 89, 2893-2900 (2017).

20. Vaaje-Kolstad, G., Horn, S. J., Sørlie, M. \& Eijsink, V. G. H. The chitinolytic machinery of Serratia marcescens - a model system for enzymatic degradation of recalcitrant polysaccharides. FEBS J. 280, 3028-3049 (2013).

21. Weikert, T., Niehues, A., Cord-Landwehr, S., Hellmann, M. J. \& Moerschbacher, B. M. Reassessment of chitosanase substrate specificities and classification. Nat. Commun. 8, 1698 (2017). 

1975-1986 (2020).

23. Gubaeva, E. et al. 'Slipped Sandwich' Model for Chitin and Chitosan Perception in Arabidopsis. Mol. Plant-Microbe Interact. 31, 1145-1153 (2018).

24. Shinya, T., Nakagawa, T., Kaku, H. \& Shibuya, N. Chitin-mediated plant-fungal interactions: catching, hiding and handshaking. Curr. Opin. Plant Biol. 26, 64-71 (2015).

25. Petutschnig, E. K., Jones, A. M. E., Serazetdinova, L., Lipka, U. \& Lipka, V. The Lysin Motif Receptor-like Kinase (LysM-RLK) CERK1 Is a Major Chitin-binding Protein in Arabidopsis thaliana and Subject to Chitin-induced Phosphorylation. J. Biol. Chem. 285, 28902-28911 (2010).

Grifoll-Romero, L., Pascual, S., Aragunde, H., Biarnés, X. \& Planas, A. Chitin Deacetylases: Structures, Specificities, and Biotech Applications. Polymers (Basel). 10, 352 (2018).

27. Wattjes, J. et al. Enzymatic Production and Enzymatic-Mass Spectrometric Fingerprinting Analysis of Chitosan Polymers with Different Nonrandom Patterns of Acetylation. J. Am. Chem. Soc. 141, 3137-3145 (2019).

28. Martinou, A., Bouriotis, V., Stokke, B. T. \& Vårum, K. M. Mode of action of chitin deacetylase from Mucor rouxii on partially N-acetylated chitosans. Carbohydr. Res. 311, 71-78 (1998).

29. Harmsen, R. A. G., Tuveng, T. R., Antonsen, S. G., Eijsink, V. G. H. \& Sørlie, M. Can we make Chitosan by Enzymatic Deacetylation of Chitin? Molecules 24, 3862 (2019).

30. Hoßbach, J. et al. A chitin deacetylase of Podospora anserina has two functional chitin binding domains and a unique mode of action. Carbohydr. Polym. 183, 1-10 (2018)

31. Cord-Landwehr, S., Melcher, R. L. J., Kolkenbrock, S. \& Moerschbacher, B. M. A chitin deacetylase from the endophytic fungus Pestalotiopsis sp. efficiently inactivates the elicitor activity of chitin oligomers in rice cells. Sci. Rep. 6, 38018 (2016).

32. Naqvi, S. et al. A recombinant fungal chitin deacetylase produces fully defined chitosan oligomers with novel patterns of acetylation. Appl. Environ. Microbiol. 82, 6645-6655 (2016).

33. Hembach, L., Cord-Landwehr, S. \& Moerschbacher, B. M. Enzymatic production of all fourteen partially acetylated chitosan tetramers using different chitin deacetylases acting in forward or reverse mode. Sci. Rep. 7, 17692 (2017).

34. Tokuyasu, K., Ono, H., Hayashi, K. \& Mori, Y. Reverse hydrolysis reaction of chitin deacetylase and enzymatic synthesis of $\beta$-d-GlcNAc-( $1 \rightarrow 4)-G l c N$ from chitobiose. Carbohydr. Res. 322, 26-31 (1999).

35. Rinaudo, M., Pavlov, G. \& Desbrières, J. Influence of acetic acid concentration on the solubilization of chitosan. Polymer (Guildf). 40, 7029-7032 (1999).

36. Calvo, P., Remuñán-López, C., Vila-Jato, J. L. \& Alonso, M. J. Novel hydrophilic chitosan-polyethylene oxide nanoparticles as protein carriers. J. Appl. Polym. Sci. 63, 125-132 (1997).

37. Sreekumar, S., Goycoolea, F. M., Moerschbacher, B. M. \& Rivera-Rodriguez, G. R. Parameters influencing the size of chitosan-TPP nano- and microparticles. Sci. Rep. 8, 4695 (2018).

38. Prego, C., Torres, D. \& Alonso, M. J. Chitosan nanocapsules: a new carrier for nasal peptide delivery. J. Drug Deliv. Sci. Technol. 16, 331-337 (2006).

39. Moreno, J. A. S. et al. Development of electrosprayed mucoadhesive chitosan microparticles. Carbohydr. Polym. (2018). doi:10.1016/j.carbpol.2018.02.062

40. Sreekumar, S., Lemke, P., Moerschbacher, B. M., Torres-Giner, S. \& Lagaron, J. M. Preparation and optimization of submicron chitosan capsules by water-based electrospraying for food and bioactive packaging applications. Food Addit. Contam. - Part A Chem. Anal. Control. Expo. Risk Assess. 34, 1795-1806 (2017).

41. Morris, E. R. Rheology of hydrocolloids. in Gums and Stabilisers for the Food Industry 2 (eds. Phillips, G. O., Wedlock, D. J. \& Williams, P. A.) 57-78 (1984).

42. Cox, W. P. \& Merz, E. H. Correlation of dynamic and steady flow viscosities. J. Polym. Sci. 28, 619-622 (1958).

43. Philippova, O. E. et al. Aggregation of some water-soluble derivatives of chitin in aqueous solutions: Role of the degree of acetylation and effect of hydrogen bond breaker. Carbohydr. Polym. 87, 687-694 (2012).

44. Novoa-Carballal, R., Riguera, R. \& Fernandez-Megia, E. Chitosan hydrophobic domains are favoured at low degree 
of acetylation and molecular weight. Polymer (Guildf). 54, 2081-2087 (2013).

45. Kong, M., Chen, X. G., Xing, K. \& Park, H. J. Antimicrobial properties of chitosan and mode of action: A state of the art review. Int. J. Food Microbiol. 144, 51-63 (2010).

46. Verlee, A., Mincke, S. \& Stevens, C. V. Recent developments in antibacterial and antifungal chitosan and its derivatives. Carbohydr. Polym. 164, 268-283 (2017).

47. Fukamizo, T., Honda, Y., Goto, S., Boucher, I. \& Brzezinski, R. Reaction mechanism of chitosanase from Streptomyces sp. N174. Biochem. J. 311, 377-383 (1995).

48. Horn, S. J. et al. Endo/exo mechanism and processivity of family 18 chitinases produced by Serratia marcescens. FEBS J. 273, 491-503 (2006).

49. Eide, K. B., Lindbom, A. R., Eijsink, V. G. H., Norberg, A. L. \& Sørlie, M. Analysis of productive binding modes in the human chitotriosidase. FEBS Lett. 587, 3508-3513 (2013).

50. Eide, K. B. et al. Human Chitotriosidase-Catalyzed Hydrolysis of Chitosan. Biochemistry 51, 487-495 (2012).

51. Aiba, S. Studies on chitosan: 4. Lysozymic hydrolysis of partially N-acetylated chitosans. Int. J. Biol. Macromol. 14, 225-228 (1992).

52. Nordtveit, R. Degradation of partially N-acetylated chitosans with hen egg white and human lysozyme. Carbohydr. Polym. 29, 163-167 (1996).

53. Stokke, B. T., Vårum, K. M., Holme, H. K., Hjerde, R. J. N. \& Smidsrød, O. Sequence specificities for lysozyme depolymerization of partially N -acetylated chitosans. Can. J. Chem. 73, 1972-1981 (1995).

54. Kolonko, A. K. et al. Capsaicin-Loaded Chitosan Nanocapsules for wtCFTR-mRNA Delivery to a Cystic Fibrosis Cell Line. Biomedicines 8, 364 (2020).

55. Kolonko, A. K., Bangel-Ruland, N., Goycoolea, F. M. \& Weber, W.-M. Chitosan Nanocomplexes for the Delivery of ENaC Antisense Oligonucleotides to Airway Epithelial Cells. Biomolecules 10, 553 (2020).

56. Fernández Fernández, E., Santos-Carballal, B., Weber, W.-M. \& Goycoolea, F. M. Chitosan as a non-viral cotransfection system in a cystic fibrosis cell line. Int. J. Pharm. 502, 1-9 (2016).

57. Strand, S. P. et al. Molecular design of chitosan gene delivery systems with an optimized balance between polyplex stability and polyplex unpacking. Biomaterials 31, 975-987 (2010).

58. Bellich, B., D’Agostino, I., Semeraro, S., Gamini, A. \& Cesàro, A. “The Good, the Bad and the Ugly” of Chitosans. Mar. Drugs 14, 99 (2016).

59. Younes, I. \& Rinaudo, M. Chitin and Chitosan Preparation from Marine Sources. Structure, Properties and Applications. Mar. Drugs 13, 1133-1174 (2015).

60. Lavertu, M., Darras, V. \& Buschmann, M. D. Kinetics and efficiency of chitosan reacetylation. Carbohydr. Polym. 87, 1192-1198 (2012).

61. Schatz, C., Viton, C., Delair, T., Pichot, C. \& Domard, A. Typical Physicochemical Behaviors of Chitosan in Aqueous Solution. Biomacromolecules 4, 641-648 (2003).

62. Gawkowska, D., Cybulska, J. \& Zdunek, A. Structure-Related Gelling of Pectins and Linking with Other Natural Compounds: A Review. Polymers (Basel). 10, 762 (2018).

63. Liners, F., Thibault, J.-F. \& Van Cutsem, P. Influence of the Degree of Polymerization of Oligogalacturonates and of Esterification Pattern of Pectin on Their Recognition by Monoclonal Antibodies. Plant Physiol. 99, 1099-1104 (1992).

64. Limberg, G. et al. Analysis of different de-esterification mechanisms for pectin by enzymatic fingerprinting using endopectin lyase and endopolygalacturonase II from A. Niger. Carbohydr. Res. 327, 293-307 (2000).

65. Osorio, S. et al. Partial demethylation of oligogalacturonides by pectin methyl esterase 1 is required for eliciting defence responses in wild strawberry (Fragaria vesca). Plant J. 54, 43-55 (2007).

66. Stopschinski, B. E. et al. Specific glycosaminoglycan chain length and sulfation patterns are required for cell uptake of tau versus $\alpha$-synuclein and $\beta$-amyloid aggregates. J. Biol. Chem. 293, 10826-10840 (2018).

67. Hembach, L., Bonin, M., Gorzelanny, C. \& Moerschbacher, B. M. Unique subsite specificity and potential natural function of a chitosan deacetylase from the human pathogen Cryptococcus neoformans. Proc. Natl. Acad. Sci. 117, 
$3551-3559$ (2020).

68. Remoroza, C., Broxterman, S., Gruppen, H. \& Schols, H. A. Two-step enzymatic fingerprinting of sugar beet pectin. Carbohydr. Polym. 108, 338-347 (2014).

69. Linhardt, R. J., Turnbull, J. E., Wang, H. M., Loganathan, D. \& Gallagher, J. T. Examination of the substrate specificity of heparin and heparan sulfate lyases. Biochemistry 29, 2611-2617 (1990).

70. Xiao, Z. et al. Heparin Mapping Using Heparin Lyases and the Generation of a Novel Low Molecular Weight Heparin. J. Med. Chem. 54, 603-610 (2011).

71. Gorzelanny, C., Pöppelmann, B., Pappelbaum, K., Moerschbacher, B. M. \& Schneider, S. W. Human macrophage activation triggered by chitotriosidase-mediated chitin and chitosan degradation. Biomaterials 31, 8556-8563 (2010).

72. de la Fuente, M. et al. Chitosan-based nanostructures: A delivery platform for ocular therapeutics. Adv. Drug Deliv. Rev. 62, 100-117 (2010).

73. Sørbotten, A., Horn, S. J., Eijsink, V. G. H. \& Vårum, K. M. Degradation of chitosans with chitinase B from Serratia marcescens. FEBS J. 272, 538-549 (2005).

74. Kohlhoff, M. et al. Chitinosanase: A fungal chitosan hydrolyzing enzyme with a new and unusually specific cleavage pattern. Carbohydr. Polym. 174, 1121-1128 (2017).

75. Muñoz, I., Rodríguez, C., Gillet, D. \& M. Moerschbacher, B. Life cycle assessment of chitosan production in India and Europe. Int. J. Life Cycle Assess. 23, 1151-1160 (2018).

76. Niehues, A., Wattjes, J., Bénéteau, J., Rivera-Rodriguez, G. R. \& Moerschbacher, B. M. Chitosan Analysis by Enzymatic/Mass Spectrometric Fingerprinting and in Silico Predictive Modeling. Anal. Chem. 89, 12602-12608 (2017).

77. Hirai, A., Odani, H. \& Nakajima, A. Determination of degree of deacetylation of chitosan by 1H NMR spectroscopy. Polym. Bull. 94, 87-94 (1991).

78. Wattjes, J., Niehues, A. \& Moerschbacher, B. M. Robust enzymatic-mass spectrometric fingerprinting analysis of the fraction of acetylation of chitosans. Carbohydr. Polym. 231, 115684 (2020).

79. Kumirska, J. et al. Determination of the pattern of acetylation of chitosan samples: Comparison of evaluation methods and some validation parameters. Int. J. Biol. Macromol. 45, 56-60 (2009).

80. Vårum, K. M., Anthonsen, M. W., Grasdalen, H. \& Smidsrød, O. 13C-N.m.r. studies of the acetylation sequences in partially N-deacetylated chitins (chitosans). Carbohydr. Res. 217, 19-27 (1991).

81. Solomon, O. F. \& Ciută, I. Z. Détermination de la viscosité intrinsèque de solutions de polymères par une simple détermination de la viscosité. J. Appl. Polym. Sci. 6, 683-686 (1962).

82. Calvo, P., Remuñán-López, C., Vila-Jato, J. L. \& Alonso, M. J. Development of positively charged colloidal drug carriers: Chitosan-coated polyester nanocapsules and submicron-emulsions. Colloid Polym. Sci. 275, $46-53$ (1997).

83. Fessi, H., Puisieux, F., Devissaguet, J. P., Ammoury, N. \& Benita, S. Nanocapsule formation by interfacial polymer deposition following solvent displacement. Int. J. Pharm. 55, R1-R4 (1989).

84. Amiji, M. M. Pyrene fluorescence study of chitosan self-association in aqueous solution. Carbohydr. Polym. 26, 211213 (1995).

85. Philippova, O. E. et al. Two Types of Hydrophobic Aggregates in Aqueous Solutions of Chitosan and Its Hydrophobic Derivative. Biomacromolecules 2, 483-490 (2001). 


\section{Biotechnology-derived chitosans with non-random patterns of acetylation differ from conventional chitosans in their properties and activities}

Jasper Wattjes ${ }^{1,2, \#, \text { Sruthi Sreekumar }}{ }^{1,2,3, \#, \text { Anna Niehues }}{ }^{1, \#}$, Tamara Mengoni ${ }^{1}$, Ana C. Mendes ${ }^{2}$, Edwin R. Morris ${ }^{4}$, Francisco M. Goycoolea ${ }^{1,3}$, Bruno M. Moerschbacher ${ }^{1, *}$

${ }^{1}$ Institute for Biology and Biotechnology of Plants, University of Münster, 48143 Münster, Germany

${ }^{2}$ National Food Institute, Technical University of Denmark, 2800 Kgs. Lyngby, Denmark

${ }^{3}$ School of Food Science and Nutrition, University of Leeds, LS2 9JT, Leeds, United Kingdom

${ }^{4}$ School of Food and Nutritional Sciences, University College Cork, Cork, Ireland

\# these authors contributed equally

* corresponding author

\section{Supplementary Information}

Table S1: Intrinsic viscosity [ $\eta$ ] of chitosan samples determined in water or $0.1 \mathrm{M} \mathrm{NaCl}$ at $25^{\circ} \mathrm{C}$, inclination angle $40^{\circ}$ (subscript denotes solvent).

\begin{tabular}{|c|c|c|c|}
\hline chitosan & {$[\eta]_{\mathrm{H} 2 \mathrm{O}}\left(\mathrm{mL} \mathrm{g}^{-1}\right)$} & {$[\eta]_{\mathrm{NaCl}}\left(\mathrm{mL} \mathrm{g}^{-1}\right)$} & {[]$_{\mathrm{H} 2 \mathrm{O}} /[]_{\mathrm{NaCl}}$} \\
\hline CS.34 & 7170 & 250 & 28.68 \\
\hline CS. $33^{\mathrm{E}-\mathrm{Ac}}$ & 950 & 196 & 4.84 \\
\hline
\end{tabular}

Table S2: Structural characteristics of chitosan samples used in this study ${ }^{1}$

\begin{tabular}{|c|c|c|c|c|}
\hline chitosan & $\boldsymbol{F}_{\mathrm{A}}$ & DP & Mw (kDa) & $\begin{array}{c}\text { used in Fig. \# } \\
\text { or Table \# }\end{array}$ \\
\hline $\mathrm{CS} .14^{\mathrm{N}-\mathrm{Ac}}$ & 0.14 & 1300 & 220 & $4 \mathrm{~b}$ \\
\hline $\mathrm{CS} .28^{\mathrm{N}-\mathrm{Ac}}$ & 0.28 & - & - & $1 \mathrm{c}$ \\
\hline $\mathrm{CS} .30^{\mathrm{N}-\mathrm{Ac}}$ & 0.30 & 700 & 127 & S1c \\
\hline $\mathrm{CS} .30^{\mathrm{N}-\mathrm{Ac}-1}$ & 0.30 & 1700 & $296,3 \mathrm{c}$ & $3 \mathrm{a}$ \\
\hline $\mathrm{CS} .34^{\mathrm{N}-\mathrm{Ac}}$ & 0.34 & 700 & 125 & $\begin{array}{c}\text { 2ac, 3b, } 4 \\
\text { S2 }\end{array}$ \\
& & & & Table S1 \\
\hline
\end{tabular}




\begin{tabular}{|c|c|c|c|c|}
\hline $\mathrm{CS} .35^{\mathrm{N}-\mathrm{Ac}}$ & 0.35 & - & - & $\begin{array}{c}1 \mathrm{c} \\
\mathrm{S} 1 \mathrm{c}\end{array}$ \\
\hline $\mathrm{CS} .14^{\mathrm{E}-\mathrm{Ac}}$ & 0.14 & 200 & 29 & $\begin{array}{c}4 \mathrm{~b}, 5 \\
\mathrm{~S} 3\end{array}$ \\
\hline $\mathrm{CS} .33^{\mathrm{E}-\mathrm{Ac}}$ & 0.33 & 800 & & $\begin{array}{c}1 \mathrm{c}, 2,3,4,5 \\
\mathrm{~S} 1, \mathrm{~S} 2, \mathrm{~S} 3 \\
\text { Table S1 }\end{array}$ \\
\hline $\begin{array}{c}\mathrm{CS} .17^{\mathrm{D}-\mathrm{Ac}} \\
(\mathrm{HMC} 70 / 5)\end{array}$ & 0.17 & 200 & 29 & $\begin{array}{c}5 \\
\mathrm{~S} 3\end{array}$ \\
\hline $\begin{array}{c}\mathrm{CS} .24^{\mathrm{D}-\mathrm{Ac}} \\
(\mathrm{HMC} 79 / 100)\end{array}$ & 0.24 & 1300 & 228 & $\begin{array}{c}5 \\
\mathrm{S3}\end{array}$ \\
\hline
\end{tabular}

${ }^{1}$ In addition, a series of chitosan polymers with $F_{A} 0.14,0.23,0.29,0.39$, and 0.46 was prepared by chemical $N$ acetylation and used for Fig. 1ab and S1b.

a

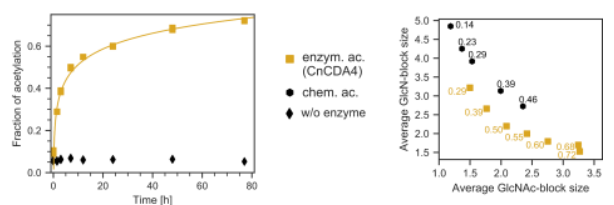

b

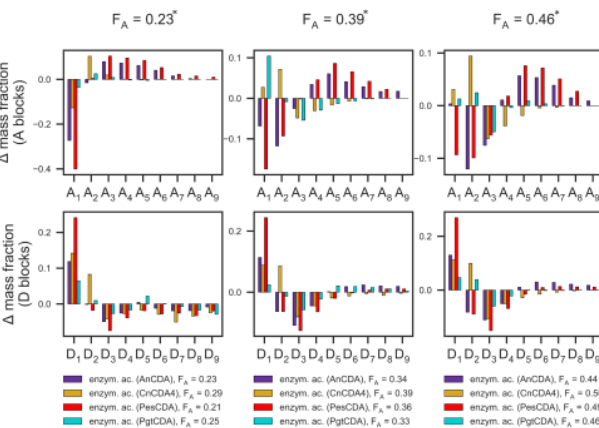

C

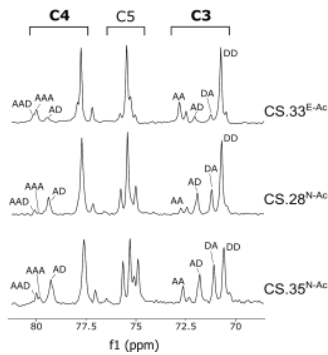

Fig. S1: a) Polyglucosamine $\left(F_{A}=0.03\right)$ was incubated in the presence of $1.5 \mathrm{M}$ sodium acetate for $76 \mathrm{~h}(\mathrm{pH} 7.5)$ with CnCDA4 from Cryptococcus neoformans or without enzyme as a control. The $F_{A}$ (left panel) and average block sizes of DP 2-10 (right panel) in the resulting chitosan polymers were analyzed using chitinosanase-based EMS fingerprinting. Chemically $N$-acetylated chitosans were used as positive controls. b) Differences of GlcNAc (A)- and GlcN (D)-block size mass fractions $\left(\boldsymbol{\Delta W}=\boldsymbol{W}_{\text {enzym. }}-\boldsymbol{W}_{\text {chem. }}\right)$ between enzymatically and chemically acetylated chitosans. ${ }^{*}, F_{A}$ of chemically $N$-acetylated chitosan to which enzymatically $N$-acetylated chitosans with similar $F_{A}$ are compared. c) Magnification of the $\mathrm{C} 4, \mathrm{C} 5$, and $\mathrm{C} 3$ region of ${ }^{13} \mathrm{C}-\mathrm{NMR}$ measurements shown in Fig. 1. (70-80 ppm). 


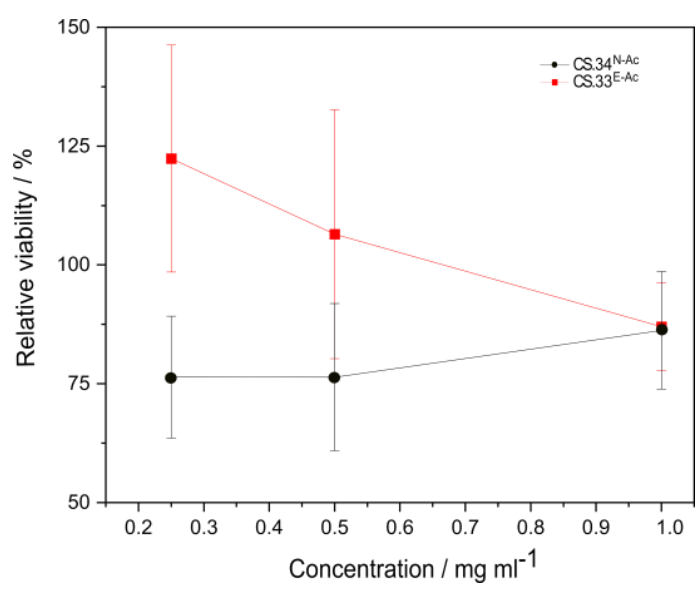

Fig. S2: In vitro cytotoxicity of enzymatically (CS.33 ${ }^{\mathrm{E}-\mathrm{Ac}}$ ) and chemically $\left(\mathrm{CS} .34^{\mathrm{N}-\mathrm{Ac}}\right) \mathrm{N}$-acetylated chitosan polymers towards HaCaT cells in 96-well plates determined using the MTT assay after $24 \mathrm{~h}$. Data are from three independent experiments with eight replicates each, plotted as mean \pm SD. 

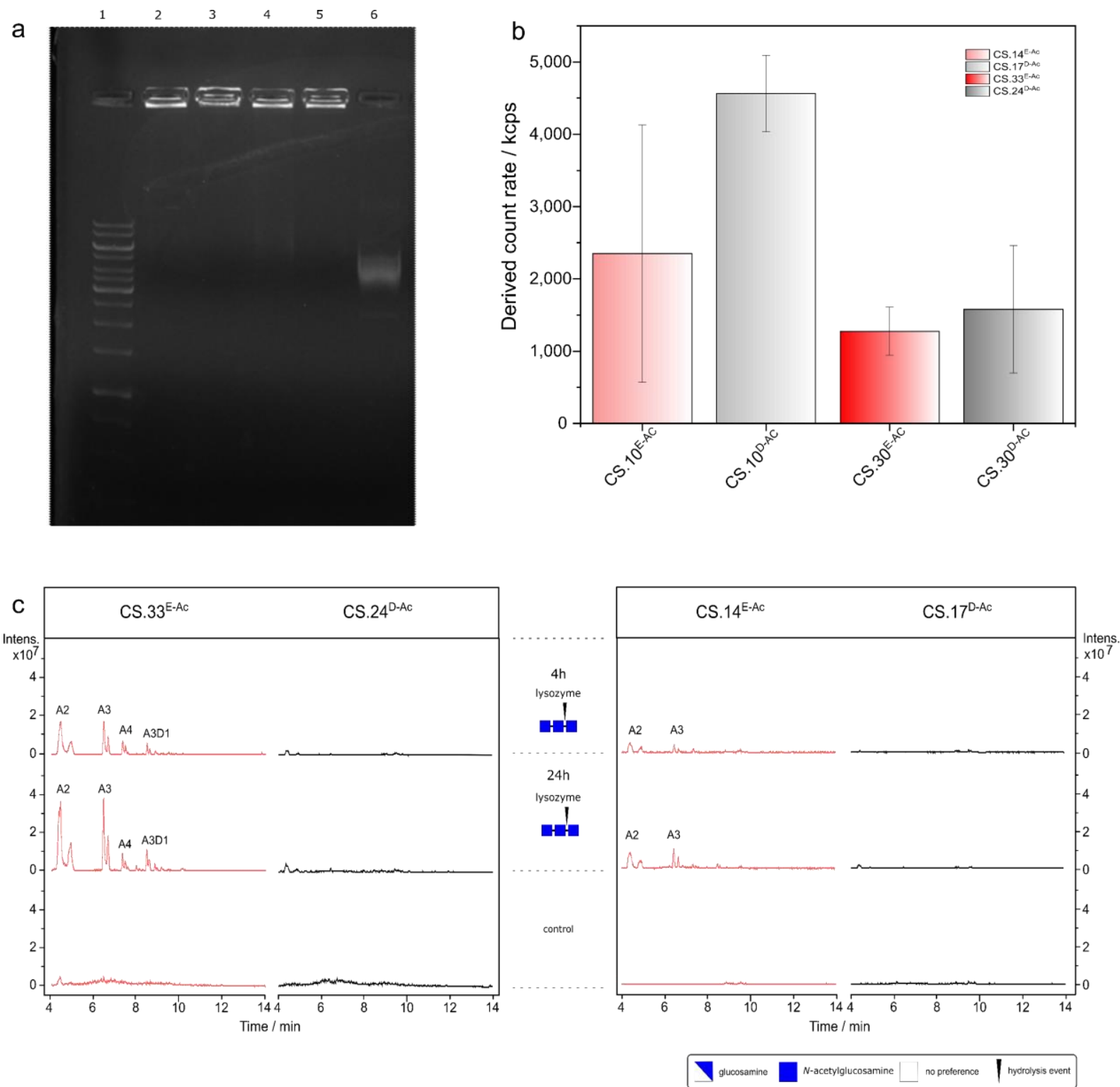

Fig S3: a) Agarose gel (1\%) electrophoresis retardation assay of chitosan-pDNA polyplexes $\left(\mathrm{NH}_{3}{ }^{+} / \mathrm{PO}_{4}^{-}\right.$molar charge ratio = 8). 1, gene ruler $1 \mathrm{~kb} ; 2$, CS.17 ${ }^{\mathrm{D}-\mathrm{Ac}}$-pDNA; 3, CS.24 ${ }^{\mathrm{D}-\mathrm{Ac}}$-pDNA; 4, CS.33 ${ }^{\mathrm{E}-\mathrm{Ac}}$-pDNA; 5, CS.14 ${ }^{\mathrm{E}-\mathrm{Ac}}$-pDNA; 6 , pDNA. b) Derived count rate in kcps measured using dynamic light scattering at $25^{\circ} \mathrm{C}$ of chitosan-pDNA polyplexes $\left(\mathrm{NH}_{3}{ }^{+} / \mathrm{PO}_{4}^{-}\right.$molar charge ratio $\left.=8\right)$. Data are from three independent experiments plotted as means \pm SD. c) Base peak chromatograms of UHPLC-ESI-MS analyses of the oligomeric hydrolysis products $(A=G I C N A C$, $\mathrm{D}=\mathrm{GlcN}$ ) of enzymatically $N$-acetylated (right parts) and chemically de- $N$-acetylated (left parts) chitosan polymers of $F_{\mathrm{A}}$ ca. 0.3 (left part) or $F_{\mathrm{A}}$ ca. 0.1 (right part) after 4 and $24 \mathrm{~h}$ of incubation with lysozyme, or in the absence of enzyme. 